\title{
Construction of an Infectious Poa semilatent virus cDNA Clone and Comparisons of Hordeivirus Cytopathology and Pathogenicity
}

\author{
Zhaolei Li, ${ }^{1}$ Zhihao Jiang, ${ }^{1}$ Xinxin Yang, ${ }^{1}$ Ning Yue, ${ }^{1}$ Xueting Wang, ${ }^{1}$ Kun Zhang, ${ }^{1}$ \\ Andrew O. Jackson, ${ }^{2}$ Dawei Li, ${ }^{1}$ and Yongliang Zhang ${ }^{1, \dagger}$
}

\author{
${ }^{1}$ State Key Laboratory of Agro-Biotechnology and Ministry of Agriculture Key Laboratory of Soil Microbiology, College of Biological \\ Sciences, China Agricultural University, Beijing 100193, China \\ 2 Department of Plant and Microbial Biology, University of California-Berkeley, Berkeley, CA 94720, U.S.A. \\ Accepted for publication 2 September 2019.
}

ABSTRACT

\begin{abstract}
Poa semilatent virus (PSLV), Lychnis ringspot virus (LRSV), and Barley stripe mosaic virus (BSMV) are members of the genus Hordeivirus in the family Virgaviridae. However, the biological properties and molecular genetics of PSLV have not been compared with other hordeiviruses. Here, we have constructed an infectious cDNA clone of the PSLV Canadian strain and provided evidence that PSLV differs from BSMV and LRSV. First, unlike the other two hordeiviruses that replicate in chloroplasts, PSLV induces dramatic structural changes in peroxisome during its infection in barley. The $\alpha$ a replication protein also localizes to peroxisomes, suggesting that PSLV replication occurs in peroxisomes. Second, PSLV encodes a $\gamma \mathrm{b}$ protein that shares 19 to $23 \%$ identity with those of other hordeiviruses, and its activity as a viral suppressor of RNA (VSR) silencing is distinct from those of BSMV and LRSV. Substitution of the BSMV $\gamma \mathrm{b}$ protein with that of PSLV or LRSV revealed a negative correlation between VSR activity and
\end{abstract}

symptom severity of the recombinant BSMV derivatives. Intriguingly, the Ser-Lys-Leu (SKL) peroxisome-targeting signals differ among $\gamma \mathrm{b}$ proteins of various hordeiviruses, including some BSMV strains. The presence of the C-terminal SKL motif in the $\gamma b$ protein impairs its silencing suppressor activity and influences symptoms. Finally, we developed a PSLV-based virus-induced gene silencing vector that induced strong and effective silencing phenotypes of endogenous genes in barley, wheat, and millet. Our results shed new light on hordeivirus pathogenesis and evolution, and provide an alternative tool for genomics studies of model hosts and economically important monocots.

Keywords: cytopathology, infectious hordeivirus clone, pathogenicity, Poa semilatent virus, SKL peroxisome-targeting signal, virus-induced gene silencing, $\gamma \mathrm{b}$ silencing suppressor
Hordeiviruses are rigid rod-shaped RNA viruses that consist of four definitive species: Barley stripe mosaic virus (BSMV), Poa semilatent virus (PSLV), Lychnis ringspot virus (LRSV), and Anthoxanthum latent blanching virus (ALBV) (Jackson et al. 2009). PSLV is composed of three plus-strand genomic (g) RNAs designated $\alpha, \beta$, and $\gamma$ that are predicted to encode seven proteins (Fig. 1A) (Hunter et al. 1986; Savenkov et al. 1998). RNA $\alpha$ encodes the $\alpha$ a protein, which is the "helicase" subunit of the viral RNAdependent RNA polymerase ( $\mathrm{RdRp}$ ) complex. RNA $\beta$ encodes the coat protein (CP) and three triple gene block (TGB) proteins designated TGB1, TGB2, and TGB3 that function in cell-to-cell and long-distance movement (Verchot-Lubicz et al. 2010). RNA $\gamma$ encodes the $\gamma$ a protein, which is the "polymerase" subunit of the $\mathrm{RdRp}$, and the $\gamma \mathrm{b}$ protein, which functions as a viral suppressor of RNA silencing (VSR) (Jackson et al. 2009; Solovyev et al. 1999).

†Corresponding author: Y. Zhang; cauzhangyl@ cau.edu.cn

Funding: This work was financially supported by the Transgenic Research Program of China (grant number 2016ZX08010-001 to Y.Z.), the National Natural Science Foundation of China (grant numbers 31830106 to D.L. and 31872637 to Y.Z.), and the Project for Extramural Scientists of SKLAB (grant number 2019SKLAB1-14).

$\mathrm{Z}$. Li and Z. Jiang contributed equally to this work.

*The $e$-Xtra logo stands for "electronic extra" and indicates that five supplementary figures and three supplementary tables are published online.

The author(s) declare no conflict of interest.

(C) 2020 The American Phytopathological Society
PSLV was first isolated on two occasions from Poa palustris and Agropyron trachycaulum within widely separated locations within Alberta, Canada (Polák and Slykhuis 1972; Slykhuis 1972). A separate PSLV isolate from Hungary has also been used for PSLV investigations by Russian investigators (Agranovsky et al. 1992), but no biological information is available about this strain. Hence, it is possible that the virus may be widespread in native grasses throughout the world. The initial studies revealed that different isolates of PSLV have various symptoms and indicated that the virus has a broad host range, including wheat (Triticum aestivum), oats (Avena sativa), rye (Secale cereal), maize (Zea mays) and several native grass species, but not dicot species (Polák and Slykhuis 1972; Slykhuis 1972).

Electron microscopy showed that PSLV and BSMV have similar particle dimensions, and serological analyses suggested a distant relationship between the two viruses (Polák and Slykhuis 1972). Subsequent nucleic acid hybridization analyses verified that PSLV, BSMV, and LRSV are distinct but related viruses (Hunter et al. 1986; Hunter et al. 1989). Some evidence has suggested that the viruses have limited cross-protecting abilities (Polák and Slykhuis 1972). Although PSLV $\gamma \mathrm{b}$ is associated with Nicotiana benthamiana peroxisomes when expressed ectopically (Yelina et al. 2005), the replication site of PSLV has not been determined. Our previous studies indicated that BSMV and LRSV replicate in chloroplast membrane-derived structures (Jiang et al. 2018; Jin et al. 2018b; Zhang et al. 2017).

The BSMV $\gamma b$ protein is a pathogenesis determinant (Donald and Jackson 1994) and a VSR protein (Bragg and Jackson 2004; Yelina et al. 2002). The $\gamma \mathrm{b}$ protein is also multifunctional and participates directly in several biological activities in vivo, including seed transmission (Edwards 1995), replication (Zhang et al. 2017), and interference with the autophagy pathway (Yang et al. 2018a). 
Moreover, $\gamma \mathrm{b}$-mediated VSR activities and host cell death responses require phosphoprotein kinase A (PKA) phosphorylation (Zhang et al. 2018). Although the hordeivirus $\gamma b$ proteins are similar structurally, systematic determination of silencing suppressor activity and pathogenicity during PSLV infections requires genetic analyses.

A number of DNA and RNA virus vectors have been developed for virus-induced gene silencing (VIGS) to facilitate functional analyses of dicot genes in planta (Burch-Smith et al. 2004; Huang et al. 2012; Igarashi et al. 2009; Yuan et al. 2011). However, VIGS tools for analyses of monocots such as millet are limited, and infection with viruses used for VIGS may have unpredictable effects on targeted gene expression of the host. Hence, it is important for additional VIGS resources to be available for comparisons of gene targeting. PSLV infects a number of cereals, including barley, and it is noteworthy that the mild symptoms elicited by some PSLV strains provide extra advantages because the latent symptoms may ameliorate adverse effects of infection during VIGS analyses.

In this study, we constructed infectious cDNA clones of a mild Canadian PSLV isolate (designated Canadian PSLV) that was originally obtained from J.T. Slykhuis and maintained in the Jackson Lab for more than 30 years. We found that, unlike BSMV and LRSV, which replicate in association with chloroplasts, PSLV $\alpha \mathrm{a}$ and virus-like particles associate with peroxisomes, suggesting that peroxisomes may be the sites of PSLV replication. We also compared VSR activities of the three hordeivirus $\gamma \mathrm{b}$ proteins and analyzed their effects on symptom development induced by recombinant BSMV derivatives in $N$. benthamiana. Because of variations in the presence of an SKL peroxisome-targeting signal at the C-termini of hordeivirus $\gamma \mathrm{b}$ proteins, we also analyzed the effect of the SKL motif on the VSR activity of $\gamma b$ and pathogenicity of the mutant viruses. Finally, because PSLV infects several monocot crops, we developed a PSLV VIGS vector and tested its application in different Gramineae hosts.

\section{MATERIALS AND METHODS}

Plant growth conditions. $N$. benthamiana plants were grown in a climate chamber with a 16 -h light and 8-h dark photoperiod at 23 to $25^{\circ} \mathrm{C}$, as described previously ( $\mathrm{Hu}$ et al. 2015). Wheat (Yangmai 11) and barley (Yangfu 4056) were grown in a glasshouse until the one-leaf stage and then transferred to a climate chamber (Hu et al. 2015). Millet (Yugu 1 and Yuyou 206), maize (B73), sorghum (BT $\times 623$ and HN 16), and rice (Nuoyou 6211 and Nuyou 2) were grown under the same conditions as barley and wheat.

Virus purification. The Canada mild isolate of PSLV was kindly provided by Dr. J.T. Slykhuis (Ottawa Research Station, Canada Department of Agriculture, Ottawa, Canada to A.O.J.). In China, the virus was propagated in barley (Yangfu 4056), and virions were purified from emerging leaves showing obvious symptoms, as described previously (Petty et al. 1989).

5'-RACE. Viral RNAs were extracted from the purified virions with the TRIzol Reagent (Life Technologies). A common R-3'UTR primer complementary to the $3^{\prime}$-termini of viral RNAs was synthesized according to the previously published sequence (Agranovsky et al. 1992; Savenkov et al. 1998; Solovyev et al. 1996). Reverse-transcription of the viral RNAs was conducted by using $6 \mu \mathrm{g}$ of template viral RNAs and $20 \mathrm{pmol}$ of the R-3'UTR primer (Supplementary Table S1). 5'-RACE was then performed as previously described (Jiang et al. 2018) with 20 pmol of each of the primers that are listed in Supplementary Table S1. DNA fragments corresponding to the 5' -termini of the viral RNAs were digested with BamHI and EcoRI and inserted in the pGAD-T7 vector (Clontech), and DNA sequencing analyses were performed to obtain sequence information of the 5 '-ends of the PSLV RNAs. All DNA fragments were amplified using I-5 2X
High-Fidelity Master Mix (Molecular Cloning Laboratories, MCLAB).

Plasmid constructions. PSLV-CA, BSMV strain ND18, and the LRSV mild isolate were used in this study (Jiang et al. 2018; Petty et al. 1989). Based on the $5^{\prime}$-terminal sequence information obtained by $5^{\prime}$-RACE, primers corresponding to the 5 '-end of viral RNAs were designed (Supplementary Table S1). The full-length cDNA of PSLV $\alpha, \beta$, and $\gamma$ were amplified using the first-strand DNA templated as described. The PSLV $\alpha$ and $\gamma$ cDNA fragments were then cloned into pBluescript II SK (+) (Promega) using the $2 \mathrm{X}$ Seamless Master Mix (Clone Smarter) to generate the pT7-PSLV $\alpha$ and pT7-PSLV $\gamma$ plasmids. PSLV $\beta$ cDNA was inserted in the pMD19-T (TaKaRa) vector, and a T7 promoter was added immediately upstream of the $5^{\prime}$-end of the PSLV $\beta$ cDNA for the pT7-PSLV $\beta$ plasmid (Fig. 1A). The XhoI restriction site adjacent to the 3 '-terminus of the PSLV genome was used to linearize the plasmid for in vitro RNA transcription. In addition, the PSLV cDNAs were also cloned into pCB301 (Yao et al. 2011) for agroinfiltration of $N$. benthamiana. To prepare antibodies against PSLV CP, the PSLV CP gene was cloned into pET30a (+) (Novagen) to generate pET30a-CP $\mathrm{PSLV}_{\text {. }}$

For confocal analyses of the PSLV oa replication protein, the cDNA $\alpha$ a fragment was cloned into pSuper1300-GFP plasmid (Yang et al. 2010). To compare VSR activity, DNA fragments corresponding to PSLV and LRSV $\gamma b$ were PCR-amplified and cloned into the pGD vector (Goodin et al. 2002) to generate pGD$\gamma b_{\text {PSLV-SKL }}$ and pGD- $\gamma b_{\text {LRSV-SKL. The }}$ pGD- $\gamma b_{\text {BSMV-SK }}$ plasmid containing wild-type BSMV (ND18 strain) $\gamma$ b has been described in previous studies (Zhang et al. 2017). For comparison of silencing suppressor activities of the hordeivirus $\gamma \mathrm{b}$ proteins and the SKL mutants, DNA fragments corresponding to the $\gamma \mathrm{b}_{\mathrm{BSMV}-\mathrm{SKL}}$, $\gamma b_{\text {PSLV-SK }}$, and $\gamma b_{\text {LRSV-SK }}$ sequences cloned into $p G D$ vector to generate pGD- $\gamma b_{\text {BSMV-SKL }}$, pGD- $\gamma b_{\text {PSLV-SK, }}$, and pGD- $\gamma b_{\text {LRSV-SK }}$. The designation $\gamma b_{\mathrm{BSMV}}$-SKL indicates that a Leucine was added to the $C$-terminus of the BSMV $\gamma b$ protein, and $\gamma b_{\text {PSLV-SK }}$ and $\gamma b_{\text {LRSV-SK }}$ indicate that the Leucine codons at the C-termini of PSLV and LRSV $\gamma \mathrm{b}$ proteins were deleted by PCR to inactivate the SKL peroxisome coding sequence.

For comparisons of pathogenicity among different hordeivirus $\gamma b$ proteins, the pCB301-BSMV $\mathrm{PSLV}_{-\gamma \mathrm{b}}$ and pCB301-BSMV $\mathrm{LRSV}_{\mathrm{Hb}}$ plasmids were constructed from pCB301-BSMV $\gamma$ (Zhang et al. 2017) by replacing the BSMV (ND18 strain) $\gamma$ b open reading frame (ORF) with the PSLV and LRSV $\gamma b$ ORFs, respectively. To analyze the effects of tripeptide SKL on the pathogenicity of hordeiviral $\gamma b$ proteins. BSMV $\gamma \mathrm{b}_{-\mathrm{SKL}}$, PSLV $\gamma \mathrm{b}_{-\mathrm{SK}}$, and LRSV $\gamma \mathrm{b}$-sK $_{\text {sK }}$ were constructed based on the pCB301-BSMV $\gamma$, pT7-PSLV $\gamma$, and pCB301LRSV $\gamma$ mutant plasmids in which the codons encoding the Leucine residues were added to or deleted from the corresponding $\gamma \mathrm{b}$ ORFs by reverse PCR.

For development of PSLV-based VIGS derivatives, a multiple cloning site was introduced into the plasmid pT7-PSLV $\gamma$ to provide pT7-PSLV $\gamma$ MCS. Then, the 255-bp GFP (GenBank accession LC417349.1), 247-bp HvPDS (GenBank accession AY062039.1), and 205-bp HvIspH (GenBank accession AK362515.1) fragments were cloned into pT7-PSLV $\gamma$ MCS to generate $\mathrm{PSLV}_{\gamma}-\mathrm{GFP}_{255}, \mathrm{PSLV} \gamma-\mathrm{HvPDS}{ }_{247}$, and $\mathrm{PSLV} \gamma-\mathrm{HvIspH}_{205}$, respectively. All primers used for plasmid construction are listed in Supplementary Table S1, and DNA sequencing was performed to confirm the accuracy of all plasmid sequences.

Virus inoculation and agroinfiltration. All plasmids with T7 promoters were linearized with $X h o I$ before in vitro transcription. In vitro transcripts generated from pT7-PSLV $\alpha$, pT7-PSLV $\beta$, and pT7-PSLV $\gamma$ or its derivatives were mixed in a molar ratio of 1:1:1 and rub-inoculated onto leaves as described previously (Lee et al. 2012).

For the host range analysis of PSLV, barley leaves infected with PSLV were ground in $20 \mathrm{mM}$ of $\mathrm{Na}$ phosphate buffer $(\mathrm{pH} 7.2$ ) containing $1 \%$ celite, and the leaf sap was mechanically inoculated 

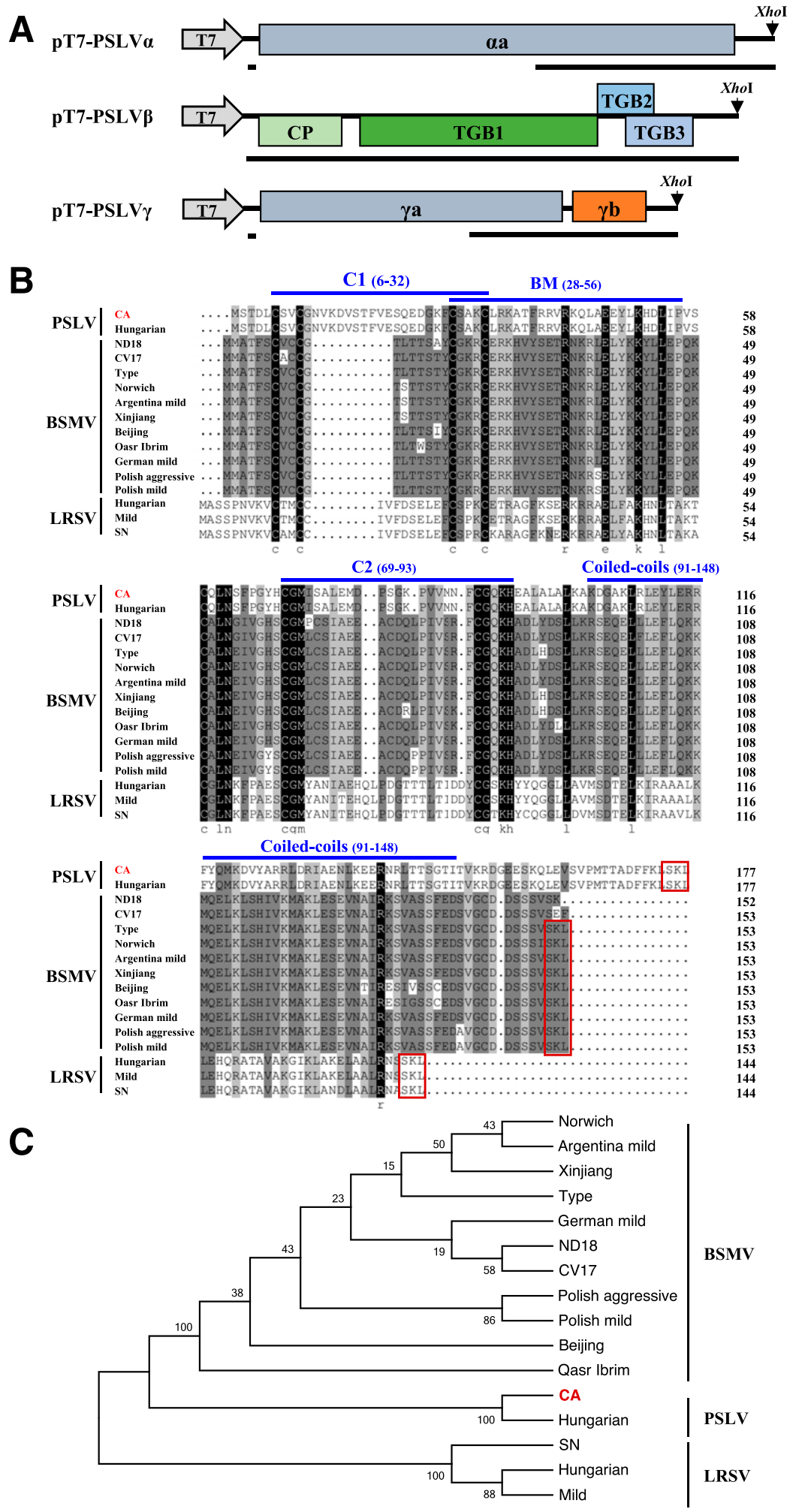

Fig. 1. Genome organization of the full-length Poa semilatent virus (PSLV) cDNA clone and sequence comparison and phylogenetic analysis of different hordeivirus $\gamma \mathrm{b}$ proteins. A, Schematic representation of the pT7-PSLV $\alpha$, pT7-PSLV $\beta$, and pT7-PSLV $\gamma$ cDNA clones. Transcription of the $\alpha, \beta$, and $\gamma$ genomic (g) RNAs is under control of the T7 promoter. The organization of the open reading frames (ORFs) of each of their RNAs is depicted. Prior to transcription, the plasmids were linearized by XhoI. Black lines beneath the cDNAs indicate sequences reported previously (Savenkov et al. 1998; Solovyev et al. 1999). B, Sequences of multiple hordeivirus $\gamma \mathrm{b}$ proteins were aligned using DNAMAN software. PSLV, Barley stripe mosaic virus (BSMV), and Lychnis ringspot virus (LRSV) strains are indicated on the left. Numbers on the right indicate the numbers of aligned $\gamma \mathrm{b}$ amino acids in each block. The predicted C1, BM, and C2 motifs and coiled-coil regions of $\gamma \mathrm{b}$ are designated by blue lines above the amino acid sequences. The blue numbers in parentheses indicate the range of corresponding motifs or regions within the PSLV $\gamma \mathrm{b}$. The $\gamma \mathrm{b}$ C-terminal SKL tripeptide is surrounded by red rectangles. The PSLV Canadian isolate investigated in this work is indicated by red font. $\mathbf{C}$, Phylogenetic tree showing relationships among various hordeivirus $\gamma \mathrm{b}$ proteins. The tree was generated by the neighbor-joining method using MEGA 5.0 software. The BSMV, PSLV, and LRSV $\gamma \mathrm{b}$ strains are shown on the right. GenBank accession numbers of different hordeivirus $\gamma \mathrm{b}$ proteins used for the construction of the phylogenetic tree are as follows: Norwich (AEP04420.1); Argentina mild (CAA36984.1); Xinjiang (AIT18341.1); Type (U13918.1); German mild (AUW36680.1); ND18 (AAA66599); CV17 (2211403B); Polish aggressive (AUW36676.1); Polish mild (AUW36678.1); Beijing (AAV54031.1); Qasr Ibrim (AHY22373.1); Hungarian (PSLV, AAB05619.1); SN (AZL87785.1); Hungarian (LRSV, YP_009508259.1); and Mild (ATO98269.1). 
onto the two-leaf stages of wheat, millet, sorghum, maize, or rice, as described previously (Yuan et al. 2011).

Agroinfiltration was conducted according to the procedures described previously (Jiang et al. 2018). To assess the VSR activities of different hordeivirus $\gamma \mathrm{b}$ proteins, A. tumefaciens cells $\left(\mathrm{OD}_{600}=0.3\right)$ harboring pGD $(\mathrm{EV})$, pGD- $\gamma b_{\mathrm{PSLV}}$, pGD- $\gamma \mathrm{b}_{\mathrm{BSMV}}$ (Zhang et al. 2017), and pGD- $\gamma \mathrm{b}_{\text {LRSV }}$ were mixed with A. tumefaciens cells $\left(\mathrm{OD}_{600}=0.4\right)$ harboring the sense GFP (sGFP) plasmid (Bragg and Jackson 2004) together with A. tumefaciens cells $\left(\mathrm{OD}_{600}=0.1\right)$ harboring the dsGFP plasmid (Johansen and Carrington 2001) and co-infiltrated in 4- to 5-week-old $N$. benthamiana leaves. The agroinfiltrated leaves were observed at 3 dpi under a long-wavelength ultraviolet (UV) lamp and photographed using a Canon EOS digital SLR camera with a yellow filter (Kodak). Similar procedures were used for tests of the VSR activities of different hordeivirus $\gamma b$ proteins in the GFP-expressing transgenic line 16c, except that the dsGFP silencing inducer was omitted from the infiltration mixture (Llave et al. 2000).

Quantitative reverse-transcription $\mathrm{PCR}$ and reversetranscription PCR. Quantitative reverse-transcription PCR (qRT-PCR) was performed according to previously described methods (Liu et al. 2012). Total RNAs were extracted from cereal leaves and measured with a NanoDrop ND-1000 analyzer (ThermoFisher Scientific). First-strand cDNA was synthesized from $6 \mu \mathrm{g}$ of total RNA by using an oligo-dT primer and M-MLV reversetranscription (Promega). The quantitative PCR experiments were performed using the 2X SsoFast EvaGreen Supermix (Bio-Rad) in the CFX96 Touch Real-Time PCR Detection System (Bio-Rad). HvActin (GenBank accession: AK356840.1), TaActin (GenBank accession: AB181991.1), and SiActin (GenBank accession: AF288226.1) sequences were chosen as internal controls for data normalization, and the results were analyzed by CFX MANGE software (Bio-Rad). All primers used for qRT-PCR are listed in Supplementary Table S2.

For RT-PCR, PSLV cDNA was prepared from purified viral RNAs by using the R-3'UTR primer and M-MLV reversetranscription (Promega). DNA fragments corresponding to PSLV RNA $\gamma$ were then PCR-amplified with the R-3'UTR and F- $\gamma 10$ primers (Supplementary Table S1).

Western blot. Western blots were performed as described previously, with minor modifications (Zhang et al. 2017). Briefly, PSLV CP ( $\left.\mathrm{CP}_{\text {PSLV }}\right)$, BSMV CP ( $\left.\mathrm{CP}_{\mathrm{BSMV}}\right)$, LRSV CP $\left(\mathrm{CP}_{\mathrm{LRSV}}\right)$, actin, and GFP antibodies were used in the relevant tests. Positive reactions were visualized by using an enhanced chemiluminescence (ECL) detection kit (GE Healthcare), and images were acquired with a ChemiDoc ${ }^{\mathrm{TM}}$ Touch Imaging System (Bio-Rad).

Expression and purification of PSLV CP protein for antibody preparation. The $\mathrm{pET} 30 \mathrm{a}-\mathrm{CP}_{\mathrm{PSLV}}$ plasmid was transformed into Escherichia coli strain Rosetta to express His-tagged fusion proteins, and the recombinant proteins were purified as described previously (Zhang et al. 2017). Briefly, the transformed bacteria were resuspended in T buffer containing $20 \mathrm{mM}$ Tris- $\mathrm{HCl}$, pH 9.0, $500 \mathrm{mM} \mathrm{NaCl}, 10 \%$ glycerol, $0.1 \%$ Triton X-100, and $1 \mathrm{mM}$ PMSF, disrupted by ultrasonication, and centrifuged for $40 \mathrm{~min}$ at $16,000 \mathrm{~g}$ to obtain the supernatants. The His-tagged PSLV protein was then purified by passage through Ni-NTA agarose affinity columns (Bio-Rad), followed by washing with increasing imidazole

TABLE 1. Comparison of the complete nucleotide (nt) sequences of Poa semilatent virus (PSLV) with those of the Lychnis ringspot virus (LRSV) mild isolate and Barley stripe mosaic virus (BSMV) strain ND18

\begin{tabular}{|c|c|c|c|c|c|}
\hline \multirow[b]{2}{*}{ Genome } & \multirow{2}{*}{$\frac{\text { PSLV }}{n t}$} & \multirow{2}{*}{$\frac{\mathrm{BSMV}}{\mathrm{nt}}$} & \multirow{2}{*}{$\frac{\mathrm{LRSV}}{\mathrm{nt}}$} & \multicolumn{2}{|c|}{ nt identity (\%) } \\
\hline & & & & PSLV/LRSV & PSLV/BSMV \\
\hline RNA $\alpha$ & 3873 & 3787 & 3759 & 56.40 & 61.74 \\
\hline RNA $\beta$ & 3608 & 3239 & 2997 & 37.60 & 54.39 \\
\hline RNA $\gamma$ & 3169 & 2790 & 2684 & 47.35 & 50.08 \\
\hline
\end{tabular}

concentration gradients. SDS-PAGE was performed to evaluate the purity and yield of the His-tagged CP, and the purified PSLV CP protein was sent to the Beijing Protein Innovation Company for antibody preparation.

Confocal laser scanning microscopy and transmission electron microscopy. A. tumefaciens cells $\left(\mathrm{OD}_{600}=0.5\right)$ harboring pSuper 1300- $\alpha \mathrm{a}-\mathrm{GFP}$ and $A$. tumefaciens cells containing $0.3 \mathrm{OD}_{600}$ of the px-rk plasmid (Nelson et al. 2007) were mixed and co-infiltrated in abaxial leaves of 4- to 5-week-old $N$. benthamiana leaves. Tissue samples were excised from $N$. benthamiana leaves at $3 \mathrm{dpi}$, and confocal analyses were performed with a Zeiss LSM-710 confocal microscope. GFP, mCherry, or chlorophyll autofluorescence was observed with $488 \mathrm{~nm}, 543 \mathrm{~nm}$, or $633 \mathrm{~nm}$ filters with an argon laser.

Tissue samples were excised from barley leaves with mosaic symptoms, and transmission electron microscopy (TEM) analyses were performed as described previously (Jin et al. 2018b). Purified PSLV virions were negatively stained with $2 \%$ uranyl acetate and observed by TEM as described previously (Hunter et al. 1986).

\section{RESULTS}

Sequence comparisons among BSMV, LRSV, and PSLV. DNA sequencing of each PSLV cDNA clone was performed, and the sequence information was deposited into GenBank with accession numbers MK377386, MK377387, and MK377388 for RNAs $\alpha$ (3873 nt), $\beta$ (3608 nt), and $\gamma$ (3169 nt), respectively (Table 1). We compared these sequences with those of the partial sequences uploaded to EMBL for the PSLV Hungarian strain (Fig. 1A). In Figure 1A, the underlined regions show PSLV RNA $\alpha$ (Z46352) and RNA $\gamma$ (M81487) sequences available in EMBL, which represent only approximately 41 and $48 \%$ of the RNA $\alpha$ and RNA $\gamma$ sequences described previously (Savenkov et al. 1998; Solovyev et al. 1996). Our comparisons with the PSLV Canadian strain revealed that high nucleotide (nt) sequence identities (>99\%) are shared with each of the deposited Hungarian gRNA sequences $($ RNA $\alpha$ nts 2135 to $3738=99.4 \%$ identity; RNA $\beta$ nts 15 to $3608=$ $99.75 \%$ identity; and RNA $\gamma 1651$ to $3169=99.87 \%$ identity). These comparisons strongly suggested that the European and North American isolates have not undergone substantial divergence despite previous reports that different North American isolates vary in their disease phenotypes (Polák and Slykhuis 1972; Slykhuis 1972).

Our comparisons of the full-length sequences of the PSLV Canada strain with those of the ND18 BSMV strain and LRSV indicated that PSLV has a slightly larger genome than BSMV and LRSV (Table 1), and that PSLV shares higher sequence identity with BSMV than with LRSV at both the nucleotide sequence level and the amino acid sequences of each ORF, indicating that PSLV is phylogenetically closer to BSMV than LRSV (Tables 1 and 2). The results also showed that each PSLV gRNA is larger than the BSMV counterparts and, similarly, that BSMV has larger gRNAs than those of LRSV (Table 1). The largest difference is that PSLV RNA $\beta$

TABLE 2. Sequence alignment of Poa semilatent virus (PSLV) open reading frames (ORFs) with those of the Lychnis ringspot virus (LRSV) mild isolate and Barley stripe mosaic virus (BSMV) strain ND18 ${ }^{\mathrm{a}}$

\begin{tabular}{|c|c|c|c|c|c|}
\hline \multirow[b]{2}{*}{ Genome } & \multirow[b]{2}{*}{ ORFs } & \multicolumn{2}{|c|}{ nt identity (\%) } & \multicolumn{2}{|c|}{ aa identity (\%) } \\
\hline & & $\begin{array}{l}\text { PSLV/ } \\
\text { LRSV }\end{array}$ & $\begin{array}{l}\text { PSLV/ } \\
\text { BSMV }\end{array}$ & $\begin{array}{l}\text { PSLV/ } \\
\text { LRSV }\end{array}$ & $\begin{array}{l}\text { PSLV/ } \\
\text { BSMV }\end{array}$ \\
\hline RNA $\alpha$ & $\alpha a$ & 59.33 & 63.10 & 55.80 & 63.32 \\
\hline \multirow[t]{4}{*}{$\operatorname{RNA} \beta$} & $\mathrm{CP}$ & 33.17 & 58.58 & 30.85 & 53.23 \\
\hline & TGB1 & 35.97 & 44.61 & 31.36 & 40.00 \\
\hline & TGB2 & 52.17 & 65.22 & 43.38 & 70.59 \\
\hline & TGB3 & 40.56 & 57.23 & 23.64 & 44.85 \\
\hline \multirow[t]{2}{*}{ RNA $\gamma$} & $\gamma \mathrm{a}$ & 54.12 & 57.07 & 55.12 & 59.89 \\
\hline & $\gamma \mathrm{b}$ & 35.44 & 35.99 & 19.23 & 23.08 \\
\hline
\end{tabular}

a nt, nucleotide; and aa, amino acid. 
has 612 and 370 more nucleotides than LRSV and BSMV, respectively. We also noted that there was no overlap between the TGB1 and TGB2 ORFs in the PSLV Canada RNA $\beta$ (Fig. 1A), in contrast to the TGB1 and TGB2 overlaps within the LRSV and BSMV gRNAs, respectively. These results implied that expression of TGB2 may be facilitated by different mechanisms than those described for BSMV TGB2 (Johnson et al. 2003).

Because of the multifunctional properties of the $\gamma \mathrm{b}$ protein (Bragg and Jackson 2004; Bragg et al. 2004; Donald and Jackson 1994; Yelina et al. 2002), we compared the amino acid sequences of all currently available hordeivirus $\gamma \mathrm{b}$ proteins. The PSLV $\gamma \mathrm{b}$ protein is more closely related to BSMV $\gamma \mathrm{b}$ than LRSV $\gamma b$ (Fig. 1B and C). Although basic amino acid motifs (containing $\mathrm{R}$ or $\mathrm{K}$ ) exist within all hordeivirus $\gamma b$ proteins (Fig. 1B), sequences within these two regions diverge substantially among the PSLV, BSMV, and LRSV isolates (Fig. 1B and Table 2). Multiple alignments of these sequences revealed six highly conserved cysteine residues present in the $\mathrm{C} 1$ and $\mathrm{C} 2$ motifs of all three virus isolates. Previous studies have shown that the cysteine residues are critical for $\mathrm{Zn}^{2+}$ binding and pathogenesis of BSMV $\gamma \mathrm{b}$ (Bragg et al. 2004); therefore, the similarities of these residues among the PSLV and LRSV $\gamma b$ proteins are consistent with conservation of essential functions. The BSMV $\gamma b$ basic motif (BM) is required for RNA binding, and the coiled-coils domain is essential for suppression of RNA silencing (Bragg and Jackson 2004; Donald and Jackson 1996).

Although basic amino acid motifs (containing $\mathrm{R}$ or $\mathrm{K}$ ) exist within all hordeivirus $\gamma b$ proteins (Fig. 1B), amino acid sequences within these two regions diverge substantially among PSLV, BSMV, and LRSV (Fig. 1B and C and Table 2). These divergencies could lead to differences in suppressor activity and pathogenesis among the $\gamma \mathrm{b}$ proteins. Moreover, sequence alignments also reveal that PSLV $\gamma b$ shares higher sequence identity with BSMV $\gamma b$ than LRSV $\gamma b$ (Table 2). Our previous studies showed that BSMV and LRSV $\gamma b$ proteins are both associated with the chloroplast membrane sites of replication (Jiang et al. 2018; Zhang et al. 2017). However, previous studies have shown that $\gamma \mathrm{b}$ of the PSLV Hungarian strain is associated with peroxisomes (Yelina et al. 2005); therefore, we speculated that PSLV might replicate in association with peroxisomes.

Analysis of the infectivity and host range of the fulllength PSLV cDNA clones. To test infectivity, the PSLV cDNA clones were linearized by XhoI treatment, followed by in vitro transcription with $\mathrm{T} 7$ polymerase. The $\alpha, \beta$, and $\gamma$ transcripts generated from the cDNAs were mixed equally and mechanically inoculated onto two-leaf stage barley. In separate experiments, barley was also inoculated with purified PSLV virions to serve as a positive control. Plants inoculated with either the PSLV virions or in vitro transcripts derived from PSLV cDNAs developed mild chlorosis in systemically infected leaves by approximately 1 week after inoculation (data not shown); at 50 days postinoculation (dpi), symptoms were still obvious (Fig. 2A). Western blot analyses also revealed the presence of the coat protein $(\mathrm{CP})$ in systemically infected leaves inoculated with in vitro-transcribed gRNAs or with virions, whereas the $\mathrm{CP}$ was absent in leaves from mock-inoculated control plants (Fig. 2B, upper panel). RT-PCR analyses with primers derived from RNA $\gamma$ also confirmed the presence of PSLV gRNA in these leaves (Fig. 2B, bottom panel). These results demonstrated that the full-length PSLV cDNA clones are infectious.

Although previous work had shown that PSLV infects several Gramineae crop plants but is unable to infect dicot plants (Polák and Slykhuis 1972; Slykhuis 1972), we aimed to test more plants for future experiments. Therefore, several potential hosts, including selected varieties of wheat, millet, maize, sorghum, rice, and $N$. benthamiana were inoculated with leaf sap prepared from PSLVinfected barley. The upper leaves of these plants were photographed and tested by Western blotting with polyclonal antibodies against PSLV CP. Mild mosaic symptoms were evident in wheat (Yangmai 11) at 15 dpi (Fig. 2C, upper panels) and millet (Yugu 1 and Yuyou
206) at 20 dpi (Fig. 2D, upper panels). Western blot analyses further confirmed PSLV infections of these plants (Fig. 2C and D, bottom panels). Additional analyses of the infected plants also indicated high infection efficiency of PSLV in millet (Yuyou 206), wheat (Yangmai 11), and barley (Yangfu 4056) (Supplementary Table S3). In contrast, obvious symptoms were not observed and the PSLV CP was not detected in PSLV-inoculated maize (B73), sorghum (BT×623 and HN16), or rice (Nuyou 6211 and Nuyou 2) (Supplementary Fig. S1A to C).

In addition, when PSLV virions were inoculated onto $N$. benthamiana leaves, the CP was not detected by Western blot analyses of extracts from the upper uninoculated leaves at $14 \mathrm{dpi}$ (Supplementary Fig. S1D). For more extensive introduction of PSLV gRNAs into $N$. benthamiana, the full-length PSLV cDNAs were cloned into the binary vector pCB301 (Yao et al. 2011), and A. tumefaciens cells harboring the pCB301-PSLV $\alpha, \beta$, and $\gamma$ derivatives were agroinfiltrated into $N$. benthamiana leaves. Again, obvious symptoms were not observed in leaves emerging from infiltrated $N$. benthamiana plants at 14 dpi (Supplementary Fig. S1E, left panels), and PSLV CP could not be detected in these leaves by Western blotting (Supplementary Fig. S1E, right panel). These
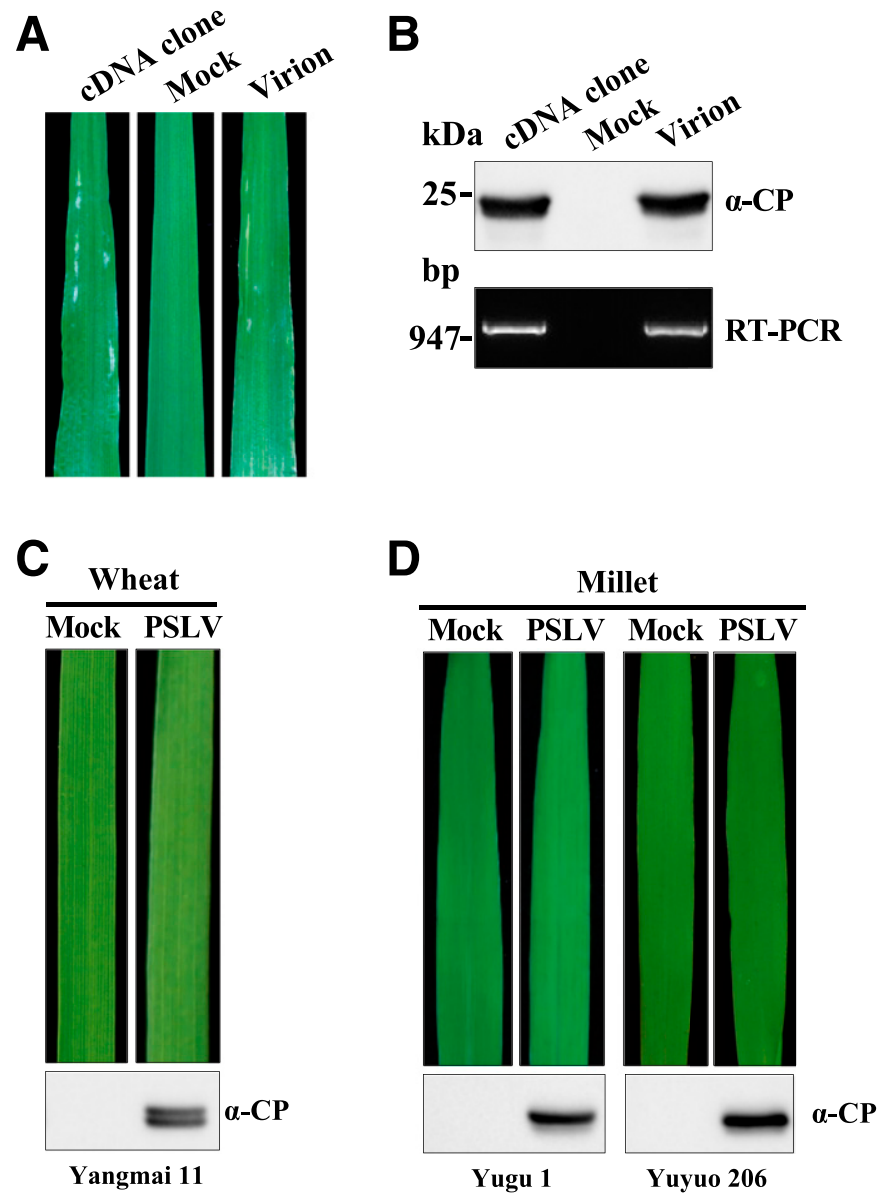

Fig. 2. Infectivity analysis of the full-length Poa semilatent virus (PSLV) cDNA clone. A, Symptoms of systemically infected leaves of barley (Yangfu 4056) at 50 days postinoculation (dpi) of PSLV in vitro transcripts, PSLV virions, or mock-inoculated barley. B, Western blots of PSLV-infected leaf extracts from the leaves described in panel A with PSLV coat protein (CP) antibodies (upper panel) and reverse-transcription PCR (RT-PCR) amplification with primers derived from PSLV RNA $\gamma$ (bottom panel). C and D, Analysis of PSLV infection in wheat and millet. Leaf sap from PSLV-infected barley leaves was rub-inoculated onto the two-leaf stage of emerged wheat and millet leaves, and the upper uninoculated leaves were photographed at $15 \mathrm{dpi}$ (upper panels). Leaves from mock-inoculated plants served as negative controls. Western blot analysis of extracts from the leaves using PSLV CP antibodies (bottom panels). The wheat and millet cultivars are designated at the bottom of each panel. 
results suggested that, unlike BSMV and LRSV, PSLV does not elicit visible or latent symptoms in N. benthamiana. In summary, our study showed that the cloned PSLV Canada isolate infects several barley, wheat, and millet cultivars, but not the tested cultivars of maize, sorghum, and rice or $N$. benthamiana.

Association of PSLV with peroxisomes. Our previous studies showed that the BSMV and LRSV $\gamma$ b proteins are targeted to chloroplasts, and that BSMV and LRSV both replicate in association with chloroplast-associated membranes (Jiang et al. 2018; Zhang et al. 2017), but the PSLV sites of replication have not been determined. However, previous experiments have shown that PSLV $\gamma \mathrm{b}$ is targeted to peroxisomes (Yelina et al. 2005); therefore, we conducted experiments to evaluate the PSLV replication site. For these analyses, we first fused GFP to the C-termini of the PSLV, BSMV, and LRSV oa replication proteins and agroinfiltrated

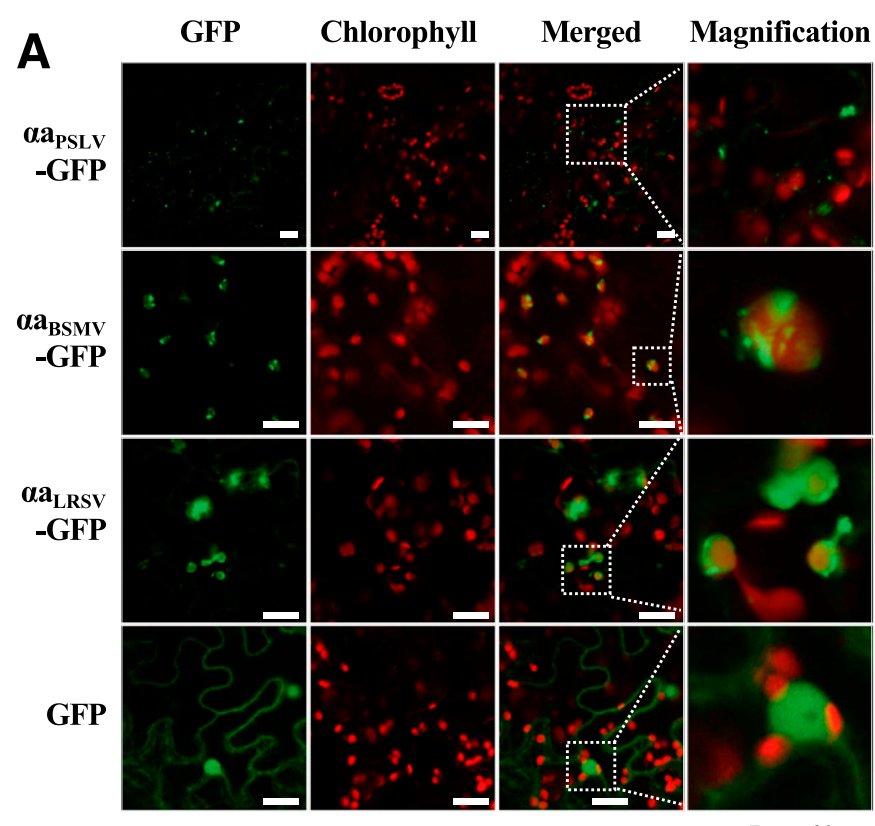

Bar $=20 \mu \mathrm{m}$

B
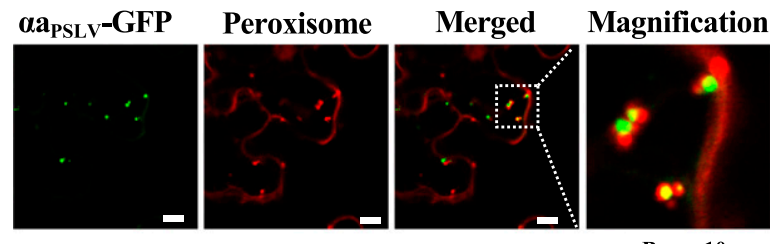

C
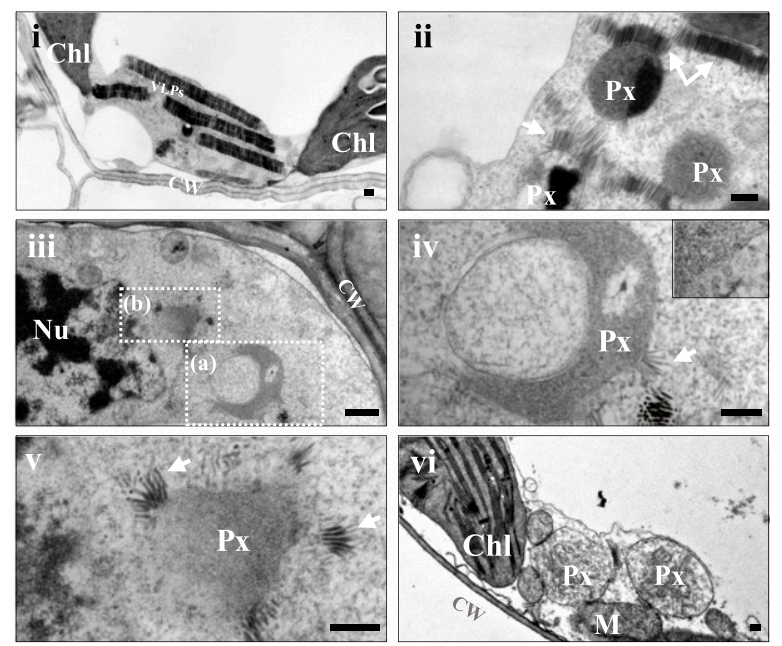

plasmids harboring the fusion proteins into $N$. benthamiana leaves. Confocal analyses of the infiltrated leaves revealed that BSMV and LRSV oa-GFP colocalized with chloroplasts, but PSLV oa-GFP did not (Fig. 3A).

To further determine the subcellular localization of PSLV oaGFP fusion protein, an mCherry-SKL fusion to provide a Cterminal tripeptide peroxisomal targeting signal was used to identify the peroxisomes (Nelson et al. 2007). We then coexpressed the mCherry-SKL fusion along with PSLV $\alpha \mathrm{a}-\mathrm{GFP}$ in $N$. benthamiana, and the resulting confocal analysis clearly showed that PSLV $\alpha \mathrm{a}$ is associated with peroxisomes in infiltrated leaves (Fig. 3B). These results provided additional evidence that PSLV replication may occur in association with peroxisomes.

To further characterize the cytopathology induced by PSLV, TEM was conducted to analyze the location of PSLV virions and the cytopathological changes in fixed sections of PSLV-infected barley cells. Our observations revealed the presence of rigid rod-shaped particles approximately $25 \mathrm{~nm}$ wide that were consistent with the width of PSLV and of varied lengths up to several hundred $\mathrm{nm}$ (Fig. $3 \mathrm{C}$ and Supplementary Fig. S2). These varied particle lengths are characteristic of purified hordeivirus particles and those in fixed tissue of infected plants (Brakke and Palomar 1976; Hunter et al. 1986; Slykhuis 1972). Peroxisomes consisting of single membranebound organelles with round to ovoid structures were also evident (Fig. 3C, panels ii and vi). A fine granular matrix that is usually present in peroxisomes and a denser area (core) with either a crystalline or an amorphous appearance was also sometimes visible (Fig. 3C, panel ii). We also observed virus-like particles (VLPs) arranged as paracrystalline structures in the cytoplasm of the PSLVinfected barley cells and as irregular aggregates around the outer membranes of the peroxisome (Fig. 3C, panels i and ii). Some peroxisomes were dilated and contained an internal hollow region suggestive of cytoplasmic invaginations in the peroxisome (Fig. 3C, panels iii and iv). In addition, small vesicles were observed at the periphery of some of the peroxisomes, which we interpreted as VLPs radiating from the vesicles (Fig. 3C, panel iv). Other peroxisomes appeared to have disintegrated or collapsed, as evidenced by dramatic alternations in their morphology, and VLPs were often clustered at the periphery of these aberrant peroxisomes (Fig. 3C, panel v). In marked contrast, leaf tissue from mockinoculated barley failed to exhibit obvious peroxisome anomalies (Fig. 3C, panel vi). These results indicated that peroxisomes are dramatically remodeled in response to PSLV infection and that the membrane anomalies may be PSLV replication sites.

Fig. 3. Peroxisome associations of Poa semilatent virus (PSLV). A, Confocal analysis of the subcellular localization of different hordeivirus $\alpha$ a proteins. Agrobacterium strains harboring the GFP-tagged $\alpha$ a replication proteins were infiltrated into $N$. benthamiana leaves, and confocal microscopy was performed at $3 \mathrm{dpi}$. A construct expressing GFP served as the control. The boxed regions in the merged panels were enlarged and are shown on the right. Chlorophyll autofluorescence is falsely colored red. Scale bars represent $20 \mu \mathrm{m}$. B, Confocal analysis of the subcellular localization of the PSLV oaGFP replication protein co-expressed with the peroxisome marker SKLmCherry (Nelson et al. 2007) in infiltrated $N$. benthamiana leaves at 3 days postinoculation (dpi). Boxed regions in the merged panel were enlarged to show the co-localization of $\alpha \mathrm{a}$ with the peroxisome. Scale bar represents $10 \mu \mathrm{m}$. C, Transmission electron microscopy (TEM) analysis of the PSLVinfected barley leaf tissues. PSLV virions were rub-inoculated onto two-leaf stage barley; at $21 \mathrm{dpi}$, systemically infected leaf tissues were excised from mosaic regions and sectioned for TEM analysis. Panels I to iii show different TEM images captured from PSLV-infected cells. Panels iv and $\mathrm{v}$ are magnifications corresponding to regions (a) and (b) in panel iii. The inset of panel iv shows a magnified view of vesicles that may be membrane-associated replication factories. Leaf tissues from mock-inoculated barley plants served as controls (panel vi). Arrows indicate the virus-like particles (VLPs). Chl, chloroplast; CW, cell wall; $\mathrm{Px}$, peroxisome; Nu, nucleus; M, mitochondria. Scale bar represents $200 \mathrm{~nm}$. 
Comparisons of $\gamma b$ silencing suppressor activity and hordeivirus replication. The $\gamma b$ protein is a viral suppressor of RNA silencing and the key pathogenicity determinant of hordeiviruses (Bragg and Jackson 2004; Jackson et al. 2009). Because of divergence between conserved amino acid sequences of different hordeivirus $\gamma \mathrm{b}$ proteins (Fig. 1B), we investigated whether the VSR activities of the $\gamma \mathrm{b}$ proteins vary among different hordeiviruses. To investigate these $\gamma \mathrm{b}$ activities, Agrobacterium harboring the empty vector $(\mathrm{EV})$ or different $\gamma \mathrm{b}$ expression cassettes, together with bacteria containing sGFP and dsGFP expression cassettes, were agroinfiltrated into $N$. benthamiana leaves. At 3 dpi, GFP fluorescence was observed under a long-wavelength UV lamp with a yellow filter. The results revealed faint GFP fluorescence in leaf regions co-infiltrated with the control EV and sGFP and dsGFP (Fig. 4A). In contrast, strong GFP fluorescence was observed in leaf regions co-infiltrated with different hordeiviral $\gamma b$ derivatives and sGFP and dsGFP (Fig. 4A). Leaves co-infiltrated with $\gamma b_{\text {BSMV }}$ sGFP and dsGFP displayed the brightest GFP fluorescence, whereas the weakest fluorescence was present in leaves co-infiltrated with the $\gamma \mathrm{b}_{\mathrm{LRSV}}$ sGFP and dsGFP and the $\gamma \mathrm{b}_{\mathrm{PSLV}}$ sGFP and dsGFP combinations resulted in intermediate fluorescence intensity (Fig. 4A). Western blot analyses of GFP accumulation in the infiltrated leaf regions corresponded to the fluorescence intensities, suggesting differences in RNA silencing suppression by the three $\gamma b$ proteins (Fig. 4B). Furthermore, when leaves of the GFP-expressing transgenic line 16c were co-infiltrated with Agrobacterium harboring the EV plasmid or different $\gamma b$ expression cassettes together with Agrobacterium containing the sGFP expression cassette (Fig. 4C and D), the results were consistent with those previously shown. These results suggested that the BSMV and PSLV $\gamma b$ proteins have more effective VSR activities in the leaf infiltration assay than the LRSV $\gamma \mathrm{b}$ protein.

To compare the effects of different $\gamma b$ derivatives on hordeivirus replication, the BSMV $\gamma \mathrm{b}$ ORF was replaced with the PSLV and LRSV $\gamma \mathrm{b}$ ORFs to generate the BSMV $\mathrm{PSLV}-\gamma \mathrm{b}_{\mathrm{b}}$ and BSMV $\mathrm{BRSV}-\gamma \mathrm{b}_{\mathrm{b}}$ hybrid viruses. $N$. benthamiana plants at the six-leaf stage were agroinfiltrated with the EV, BSMV, BSMV $\mathrm{PSLV}_{-\gamma \mathrm{b}}$, or BSMV ${ }_{\mathrm{LRSV}-\gamma \mathrm{b}}$ plasmids. At $5 \mathrm{dpi}$, the emerging leaves of $N$. benthamiana developed systemic symptoms with mild chlorotic spots after $\mathrm{BSMV}_{\mathrm{PSLV}-\gamma \mathrm{b}}$ agroinfiltration, but the leaves of plants infected with BSMV $_{\text {LRSV- } \gamma \mathrm{b}}$ exhibited only mild chlorosis (Fig. 5A). Similar leaves of plants infected with BSMV failed to develop obvious symptoms at $5 \mathrm{dpi}$, but a typical mild mosaic appeared after approximately7 dpi (Fig. 5B), and similar symptoms were present at 14 dpi (Fig. 5C). In contrast, extensive chlorosis and leaf curl symptoms were obvious in $\mathrm{BSMV}_{\mathrm{PSLV}-\gamma \mathrm{b}}$-infected leaves, and a milder and less extensive mosaic was present in leaves of BSMV $_{\text {LRSV- } \gamma \mathrm{b}}$-infected plants (Fig. 5B). By 14 dpi after agroinoculation with BSMV, the leaves had not progressed beyond a mild mosaic, but a severe mosaic interspersed with "green islands" and leaf curling symptoms appeared in the hybrid BSMV $\mathrm{PSLV} \gamma \mathrm{b}$ systemically infected leaves (Fig. 5C). In contrast, the BSMV $\mathrm{LRSV}^{-\gamma \mathrm{b}^{-}}$ infected plants developed a white leaf chlorosis, and the plants were wilted and stunted (Fig. 5C). Although the infection phenotype is not directly correlated with silence suppressor activities, the chimeric $\gamma b$ derivatives have varied effects on pathogenesis of the BSMV hybrids.

To evaluate virus accumulation of the BSMV hybrids, samples were extracted from systemically infected leaves with typical symptoms at $14 \mathrm{dpi}$, respectively, and subjected to Western blot analyses. The results also showed that the CP accumulation in leaves infected with BSMV $\mathrm{LRSV}_{-\gamma \mathrm{b}}$ was nearly twice that of BSMVinfected leaves, and that BSMV $\mathrm{PSLV}_{\mathrm{Pb}}$ had almost four-times as much $\mathrm{CP}$ as the BSMV-infected leaves (Fig. 5D). Our results indicated that the different $\gamma \mathrm{b}$ proteins differ substantially in their effects on symptom development and on replication of the BSMV hybrid viruses. These results suggested that differences in the amino acid sequences of the BSMV, PSLV, and LRSV $\gamma b$ proteins contribute to variations in VSR activities and virus accumulation.
The C-terminal SKL peroxisome-targeting motif attenuates $\gamma b$ silencing suppressor activities but enhances hordeivirus accumulation. The PSLV $\gamma b$ C-terminal SKL motif was previously shown to be required for localization of PSLV $\gamma b$ to peroxisomes and to have minimal effects on RNA silencing (Yelina et al. 2005). Our comparisons of amino acid sequences of different hordeivirus $\gamma \mathrm{b}$ proteins revealed that except for the $\gamma \mathrm{b}$ proteins of the BSMV ND18 and CV17 strains, the C-termini of all of the other sequenced hordeivirus $\gamma b$ proteins have an SKL motif (Fig. 1B; note the red rectangular box around the C-terminal SKL sequences). To obtain additional information about requirements of the SKL motif for $\gamma \mathrm{b}$ silencing suppression, we constructed BSMV ND18, PSLV, and LRSV $\gamma b$ mutants. Then, we inserted these mutants along with the wild-type $\gamma \mathrm{b}$ derivatives into A. tumefaciens. To create the $\gamma \mathrm{b}$ mutant, a leucine residue was added to the BSMV ND18 $\gamma \mathrm{b}$ Cterminus to generate an ND18 mutant $\left(\gamma b_{\text {BSMV-SKL }}\right)$ with an SKL motif. We also excised the leucine residues comprising the SKL motifs of the PSLV and LRSV $\gamma b$ sequences to engineer the $\gamma b_{\text {PSLV-SK }}$ and $\gamma \mathrm{b}_{\text {LRSV-SK }}$ mutants. Then, $N$. benthamiana leaves were infiltrated with Agrobacterium mixtures containing the sGFP and dsGFP expression cassettes and Agrobacterium strains harboring the wild-type PSLV $\gamma b\left(\gamma b_{\text {PSLV-SKL }}\right)$, BSMV ND18 $\gamma b\left(\gamma b_{\text {BSMV-SK }}\right)$,

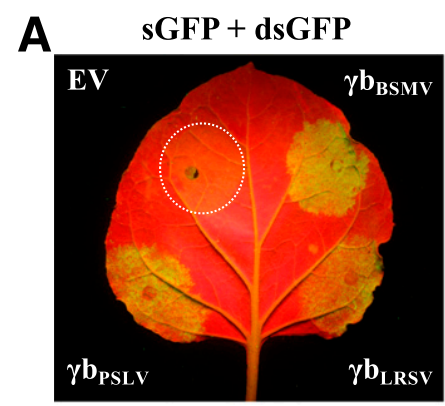

B

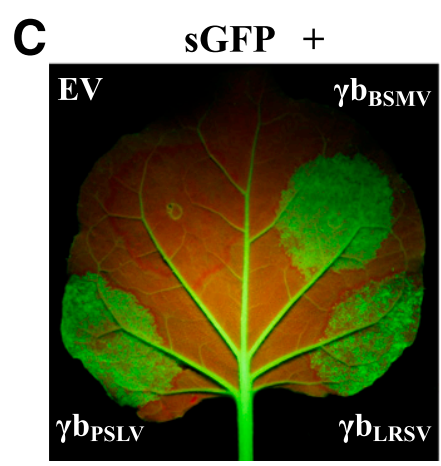

D
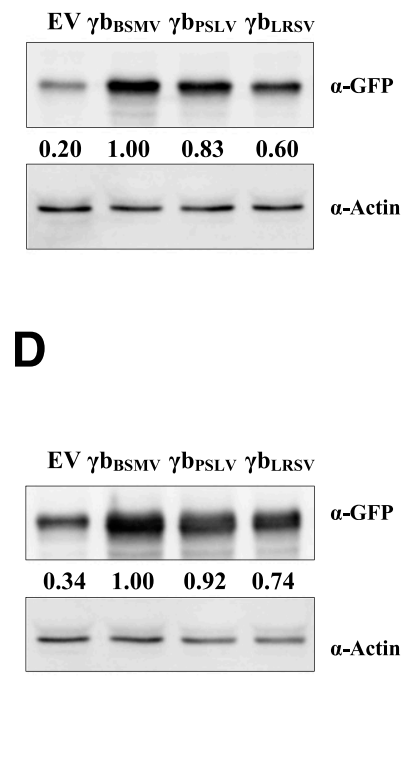

Line 16c

Fig. 4. Comparison of viral suppressor of RNA (VSR) activities of different hordeivirus $\gamma \mathrm{b}$ proteins. A, Analysis of the suppressor activities of Barley stripe mosaic virus (BSMV), Poa semilatent virus (PSLV), and Lychnis ringspot virus (LRSV) $\gamma \mathrm{b}$ proteins. Agrobacterium harboring the hordeivirus $\gamma \mathrm{b}$ expression cassettes were co-infiltrated into different regions of the leaf with Agrobacterium containing sGFP and dsGFP expression derivatives. Infiltrated leaves were photographed at 3 days postinoculation (dpi) under long-wave ultraviolet (UV) light with a yellow filter. The empty vector (EV) pGD served as a negative control. B, Western blot analysis with antibodies against GFP in the infiltrated regions indicated in A (upper panel). Equal protein loading was verified by actin protein (bottom panel). ImageJ software was used to quantify the GFP bands, and intensity of the $\gamma \mathrm{bBSMV}$ band was set to 1.00. C, Analysis of the suppressor activities of hordeivirus $\gamma \mathrm{b}$ proteins in $N$. benthamiana line 16c. Agrobacterium containing the hordeivirus $\gamma \mathrm{b}$ expression cassettes and Agrobacterium harboring the sGFP expression cassette were co-infiltrated in different regions of the same leaves of line $16 \mathrm{c}$ plants. Infiltrated leaves were photographed at 5 dpi under long-wave UV light with a yellow filter. The EV (pGD) served as a negative control. D, Western blot analysis with antibodies against GFP in the infiltrated regions $(\mathbf{C}$, upper panel). Actin protein was used to monitor protein loading (bottom panel). 
or LRSV $\gamma \mathrm{b}\left(\gamma \mathrm{b}_{\text {LRSV-SKL }}\right)$ derivatives (Fig. 6A, B, and C), as was performed by Bragg and Jackson (2004). Similarly, the sGFP and dsGFP expression cassettes were mixed with the respective mutant PSLV $\gamma \mathrm{b}\left(\gamma \mathrm{b}_{\text {PSLV-SK }}\right)$, BSMV $\left(\gamma \mathrm{b}_{\text {BSMV-SKL }}\right)$, or LRSV $\left(\gamma \mathrm{b}_{\text {LRSV-SK }}\right)$ plasmids. The EV plasmid was also mixed with the sGFP and dsGFP expression plasmids to provide an infiltration control. At 3 dpi, regions of leaves infiltrated with $\gamma b$ derivatives containing an SK C-terminus exhibited strong GFP fluorescence, whereas regions containing the C-terminal SKL motif exhibited much lower levels of fluorescence (Fig. 6A to C, upper panels).

Western blot analyses showed that GFP accumulation in the infiltrated leaf regions was consistent with the intensity of GFP fluorescence (Fig. 6A to C, bottom panels) because GFP amounts in regions infiltrated with the $\gamma \mathrm{b}_{\text {PSLV-SK }}$ and $\gamma \mathrm{b}_{\text {LRSV-SK }}$ mixtures were more than twice those of the $\gamma b_{\text {PSLV-SKL }}$ and $\gamma b_{\text {LRSV-SKL }}$ derivatives. This trend was similar but somewhat lower in BSMV infiltrations containing wt $\gamma b_{\text {BSMV-SK }}$ compared to the mutant $\gamma b_{\text {BSMV-SKL. }}$. These results differ from those of Yelina et al. 2005, and suggested that ectopically expressed $\gamma \mathrm{b}$ containing the C-terminal SKL motif attenuates VSR activities of $\gamma \mathrm{b}$.

To investigate the extent to which the $\gamma b$ SKL motif affects virus replication, the $\gamma \mathrm{b}$ ORFs of the PSLV, BSMV ND18, and LRSV RNA $\gamma$ plasmids were mutated to $\gamma \mathrm{b}_{\mathrm{PSLV}-\mathrm{SK}}, \gamma \mathrm{b}_{\mathrm{BSMV}-\mathrm{SKL}}$, and

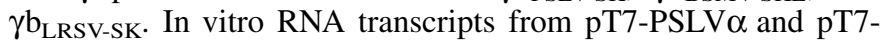
PSLV $\beta$ were first mixed with in vitro wild-type PSLV RNAs or the

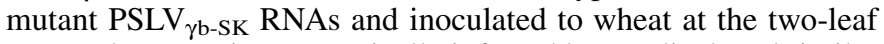
stage. The emerging systemically infected leaves displayed similar mild chlorotic symptoms at $11 \mathrm{dpi}$ in comparison with leaves from mock inoculations (Fig. 6D). However, Western blots (Fig. 6E), revealed that the wtPSLV-infected leaves contained almost twice as much CP as the mutant PSLV ${ }_{\gamma \mathrm{b}-S K}$ infections, suggesting that the $\gamma b_{\text {SKL }}$ derivative contributes more efficiently to PSLV accumulation in wheat than the $\gamma b_{\mathrm{SK}}$ derivative.

In a second set of experiments, $N$. benthamiana plants at the sixleaf stage were agroinfiltrated with the EV control, BSMV, $\mathrm{BSMV}_{\gamma \mathrm{b}-\mathrm{SKL}}, \mathrm{LRSV}$, or $\mathrm{LRSV}_{\gamma \mathrm{b}-\mathrm{SK}}$. By $14 \mathrm{dpi}$, the emerging

A

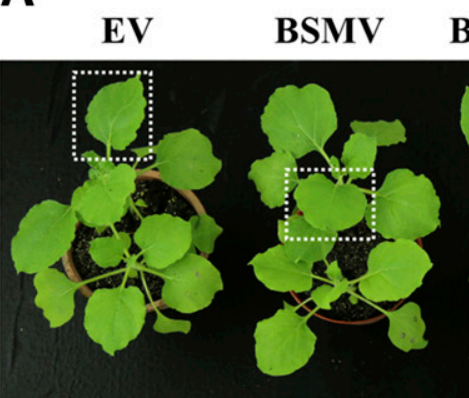

BSMV PSLV- $\gamma \mathbf{b}$

BSMV LRSV- $\gamma$ b

B
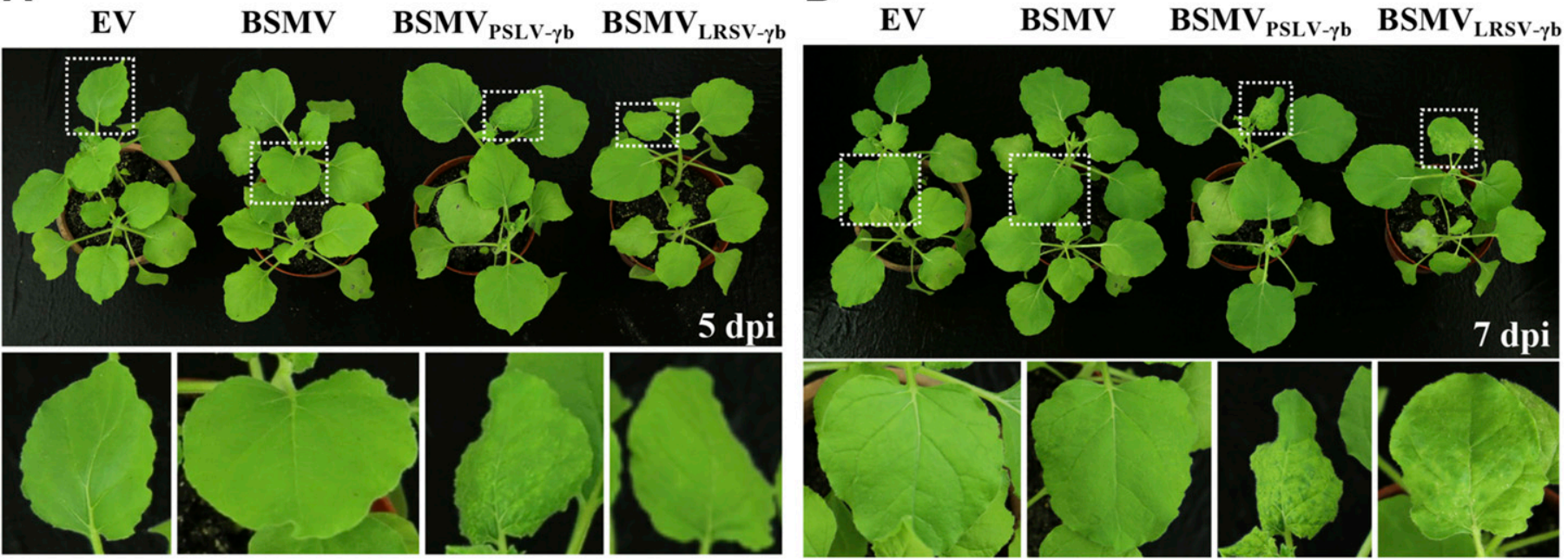

C

EV BSMV

BSMV $_{\text {PSLV- } \gamma \mathbf{b}}$

BSMV $_{\mathrm{I}}$
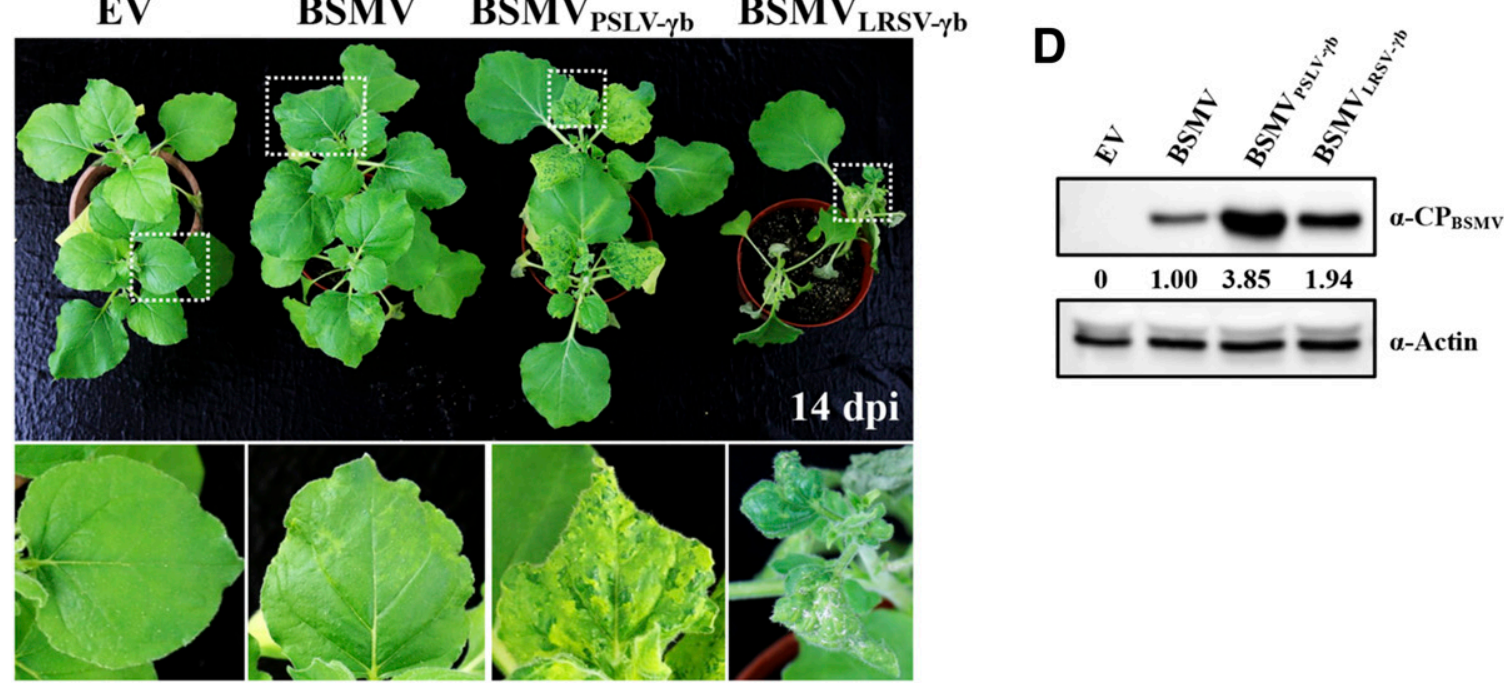

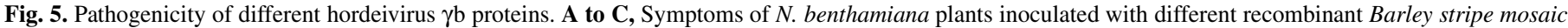

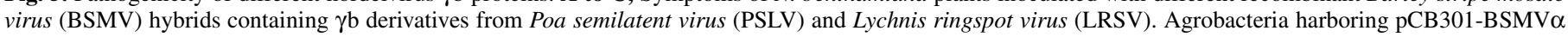

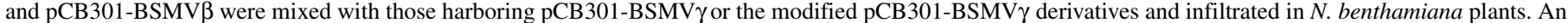

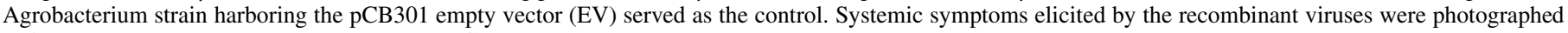

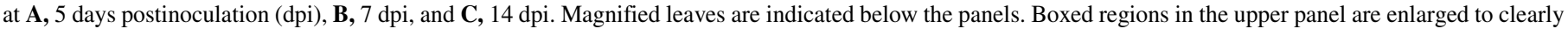

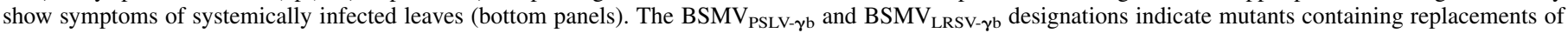

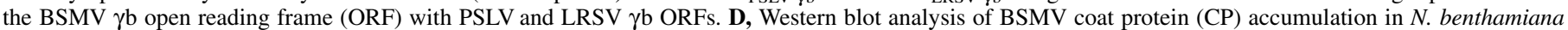

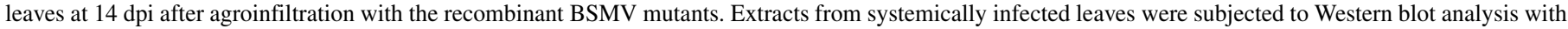
the BSMV CP antibody (upper panel). An actin antibody was used to monitor protein loading (bottom panel). 
A

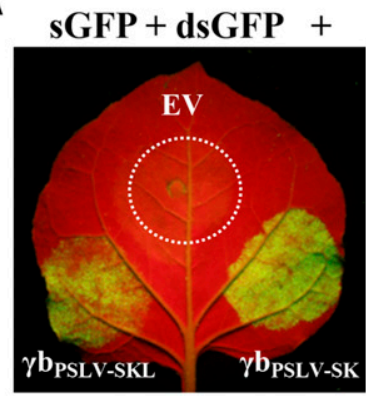

B

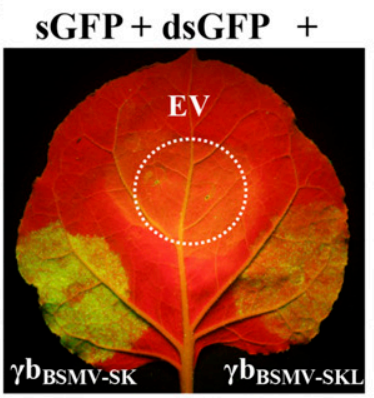

EV $\gamma \mathbf{b}_{\text {BSMV-SK }} \gamma \mathbf{b}_{\text {BSMV-SKL }}$

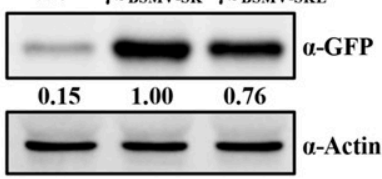

C

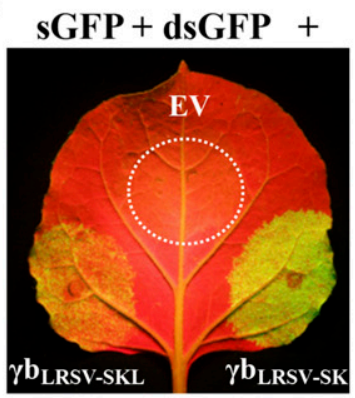

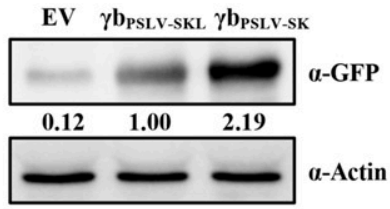

D

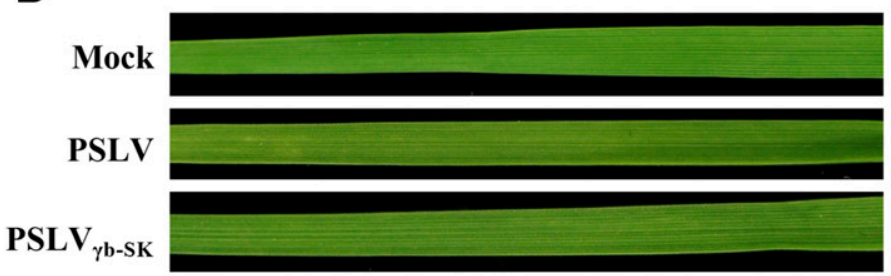

(11 dpi)

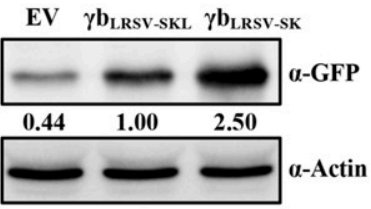

E

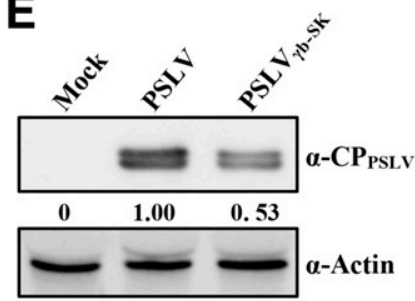

F

EV

BSMV

BSMV $_{\gamma b-S K L}$

H

EV

LRSV

$\mathbf{L R S V}_{\gamma \mathrm{b}-\mathrm{St}}$
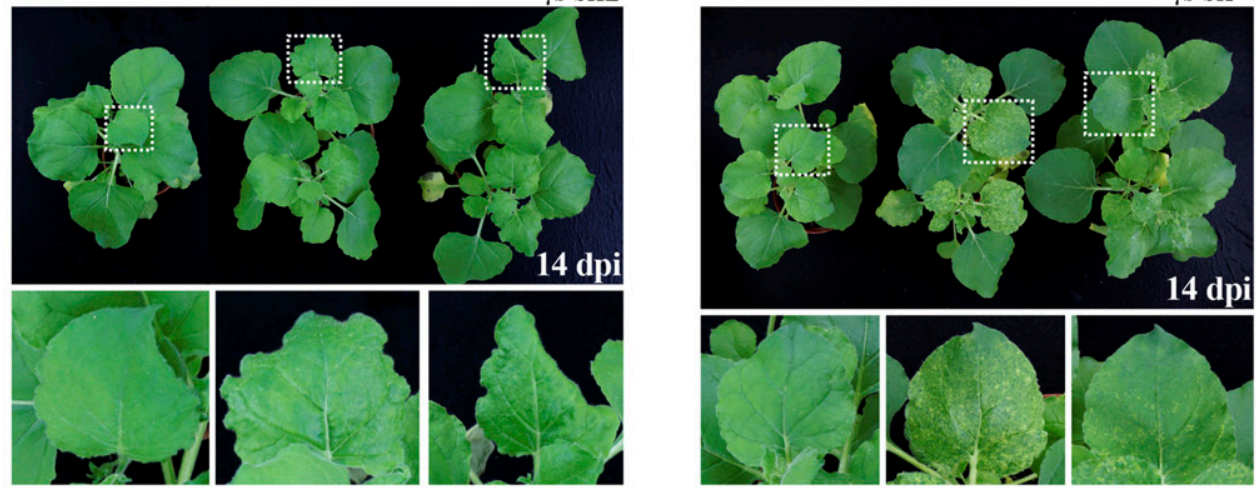

G
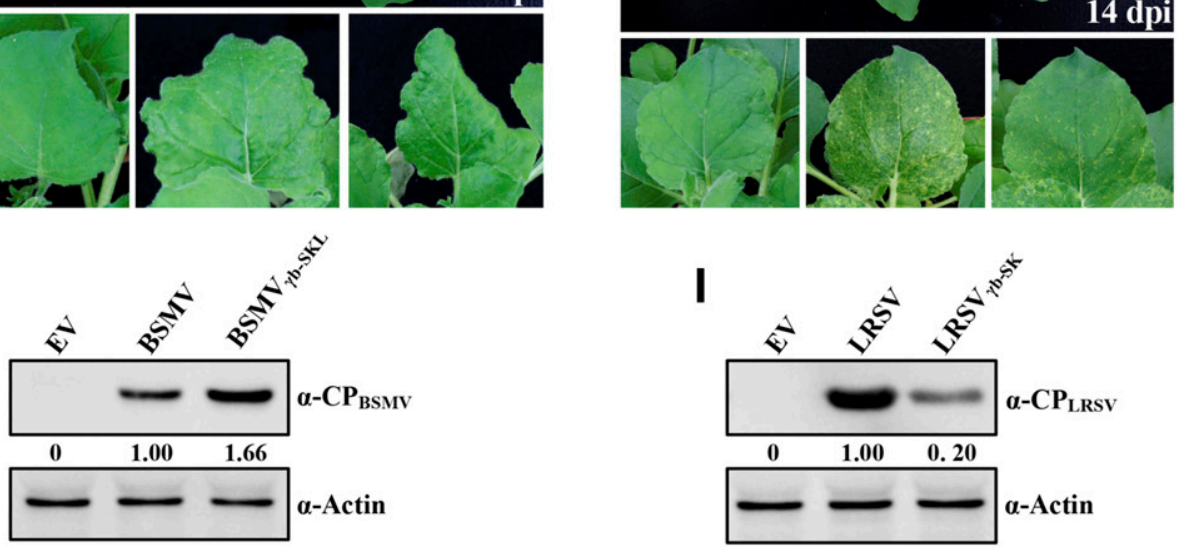

Fig. 6. Effects of the SKL peroxisome-targeting signal on the silencing suppressor activity and pathogenicity of $\gamma \mathrm{b}$ proteins. A to C, Analysis of the effects of SKL peroxisome-targeting signal mutations on $\gamma \mathrm{b}$ viral suppressor of RNA (VSR) activity. Agrobacterium harboring $\gamma \mathrm{b}$ expression cassettes or SKL mutants together with various mixtures of the sGFP expression plasmid and the dsGFP silencing inducer were co-infiltrated in different regions of the same leaf. At 3 days postinoculation (dpi), infiltrated leaves were photographed under long-wave ultraviolet (UV) light with a yellow filter; representative results are shown (top panels). The empty vector (pGD) served as a negative control. Western blot analysis of extracts from the infiltrated regions with GFP antibodies are shown (middle panels). The actin protein served as a loading control (bottom panels). The $\gamma \mathrm{b}_{\text {PSLV-SK}}, \gamma \mathrm{b}_{\mathrm{BSMV}} \mathrm{sK}_{\mathrm{S}}$, and $\gamma \mathrm{b}_{\mathrm{LRSV}-\mathrm{SK}}$ symbols indicate $\gamma \mathrm{b}$ proteins lacking the $\mathrm{C}$ terminal SKL tripeptide, whereas $\gamma b_{\text {PSLV-SKL }}, \gamma b_{\text {BSMV-SKL }}$, and $\gamma b_{\text {LRSV-SKL }}$ correspond to $\gamma b$ proteins containing the C-terminal SKL sequence. GFP accumulation was quantified by ImageJ software, and signals of the wild-type hordeivirus $\gamma \mathrm{b}$ bands were set to 1.00. D and E, Comparisons of PSLV and the PSLV SKL mutant in infected wheat. In vitro transcripts of PSLV and PSLV ${ }_{\gamma \mathrm{b}-S K}$ were mechanically inoculated on two-leaf stage wheat (Yangmai 11), and systemically infected leaves were photographed at $11 \mathrm{dpi}(\mathrm{D})$. Symptoms of $N$. benthamiana plants 14 dpi after agroinoculation with Barley stripe mosaic virus (BSMV) and F, BSMV ${ }_{\gamma \mathrm{b}-\mathrm{SKL}}$, Lychnis ringspot virus (LRSV), and $\mathbf{H}, \mathrm{LRSV}_{\mathrm{\gamma b}-\mathrm{SK}}$. Boxed regions in the upper panels were enlarged to more clearly demonstrate symptoms in systemically infected leaves (bottom panels). Extracts from the systemically infected leaves shown in $\mathbf{D}, \mathbf{F}$, and $\mathbf{H}$ were subjected to Western blot analysis using antibodies against coat proteins (CPs) of the Poa semilatent virus (PSLV), BSMV, or LRSV (E, G, and I, upper panels). Actin was used to monitor protein loading (E, G, and I, bottom panels). The PSLV, BSMV ${ }_{\gamma b-S K L}$, and LRSV designations represent viruses containing $\gamma \mathrm{b}$ proteins with the C-terminal SKL motif. PSLV ${ }_{\gamma \mathrm{b}-\mathrm{SK}}, \mathrm{BSMV}$, and LRSV $\mathrm{Sb}_{\mathrm{bK}}$ designate viruses with $\gamma \mathrm{b}$ C-terminal SK residue. 
leaves of plants infiltrated with BSMV and the mutant BSMV $\mathrm{Vb}_{\gamma \mathrm{b} \text { SK }}$ had no obvious phenotypic differences, and both leaves exhibited mild mosaic symptoms (Fig. 6F). However, the leaves infected with the $\mathrm{BSMV}_{\gamma \mathrm{b}-\mathrm{SKL}}$ mutant had approximately $50 \%$ more $\mathrm{CP}$ accumulation than BSMV leaves (Fig. 6G). Moreover, a similar but more obvious trend was evident in comparisons of $N$. benthamiana leaves systemically infected with the LRSV virus and the LRSV $_{\gamma \mathrm{b}-\mathrm{SK}}$ mutant virus at $14 \mathrm{dpi}$. In these plants, the LRSV infections had developed obvious chlorotic spots on the emerging leaves, and these were more numerous than the milder chlorotic spots on leaves infected with the mutant $\mathrm{LRSV}_{\gamma \mathrm{b}-\mathrm{SK}}($ Fig. $6 \mathrm{H})$. Moreover, differences in replication were even more striking because approximately five-fold more $\mathrm{CP}$ accumulated in leaves infected with the LRSV virus than with the $\mathrm{LRSV}_{\gamma \mathrm{b}-\mathrm{SK}}$ mutant viruses (Fig. 6I). These results indicated that all three hordeivirus strains encoding the $\gamma b_{S K L}$ motif accumulate to higher levels than their $\gamma b_{S K}$ derivatives in both monocot and dicot plants. As mentioned, we believe these results are an indirect consequence of attenuated RNA silencing suppression by the $\gamma b_{\text {SKL }}$ proteins.

Development of PSLV as a VIGS vector for barley, wheat, and millet. Because PSLV induced very mild symptoms in barley, wheat, and millet (Fig. 2A, C, and D and Supplementary Fig. S3), we developed PSLV as a VIGS vector for these species. Multiple cloning sites were inserted immediately downstream of the $\gamma \mathrm{b}$ stop codon (Fig. 7A), and the PDS (phytoene desaturase) and IspH (4-hydroxy-3-methylbut-2-enyl diphosphate reductase) genes were chosen as targets for silencing. The 247-bp cDNA PDS and the 205 bp IspH DNA fragments from barley were cloned into the pT7-PSLV $\gamma$ MCS derivative to provide the PSLV $\gamma$-HvPDS 247 and $\mathrm{PSLV}_{\gamma}$-HvIspH $\mathrm{H}_{205}$ silencing vectors. In addition, a 255-bp DNA GFP fragment was cloned into pT7-PSLV $\gamma \mathrm{MCS}$ to serve as a control. The pT7-PSLV $\gamma$ MCS gene silencing derivatives were linearized, followed by in vitro transcription; the resulting RNAs were mixed with PSLV RNA $\alpha$ and RNA $\beta$ transcripts and mechanically inoculated onto barley, wheat, and millet plants at the two-leaf stage. For convenient descriptions, mixed RNAs consisting of PSLV $\alpha$, PSLV $\beta$, and $\gamma-\mathrm{GFP}_{255}$ were designated PSLV::GFP 255 , whereas mixtures with PSLV $\alpha$, PSLV $\beta$, and $\mathrm{PSLV}_{\gamma}-\mathrm{HvPDS}_{247}$ or PSLV $\gamma$-HvIspH ${ }_{205}$ RNAs were designated PSLV::HvPDS 247 or PSLV::HvIspH $H_{205}$.

Western blot analyses with PSLV CP antibodies confirmed PSLV::GFP ${ }_{255}$, PSLV::HvPDS ${ }_{247}$, and PSLV::HvIspH $H_{205}$ infections of each of the inoculated hosts and revealed robust $\mathrm{CP}$ expression (Supplementary Fig. S4). At 15 dpi, photobleaching phenotypes appeared as streaks associated with the vasculature of emerging leaves of barley, wheat, and millet plants inoculated with PSLV:: $H v P D S_{247}$, whereas mock-inoculated or PSLV::GFP 255 -inoculated plants failed to develop photobleaching (Fig. 7B, D, and F, left panels). The qRT-PCR analyses indicated that the PDS mRNA levels in photobleached leaves were reduced by approximately $80 \%$ in barley and wheat and by approximately $50 \%$ in millet compared with control plants inoculated with PSLV::GFP 255 (Fig. 7C, E, and $\mathrm{G})$. Only wheat and barley plants inoculated with PSLV::HvIspH $\mathrm{H}_{205}$ developed a bleached phenotype in the systemically infected leaves (Fig. 7B and D), but obvious phenotypic differences were not observed in PSLV::HvIspH $H_{205}$-inoculated and PSLV::GFP $255^{-}$ inoculated millet plants (Fig. 7F). Moreover, qRT-PCR analyses consistently revealed significant reductions in $I s p H$ mRNA levels in both wheat and barley, but not in millet (Fig. 7C, 7E, and 7G). Possible reasons for the $I s p H$ differential silencing effects on millet are discussed here. Together, these results demonstrated that PSLVbased VIGS can be an effective tool for gene silencing in barley, wheat, and millet.

\section{DISCUSSION}

Comparative analyses of different viruses, especially those in the same genus, enhance our understanding of virus evolution and molecular mechanisms underlying pathogenesis. Although previous serological analyses and nucleotide acid hybridization have indicated that BSMV is more closely related to PSLV than to LRSV (Hunter et al. 1989; Savenkov et al. 1998), definitive evidence has not been available because the complete PSLV genome sequence had not been deposited in sequence databases. In this study, we have compared the full-length genome sequences of BSMV and LRSV with our PSLV Canada sequence, and these results confirmed that PSLV is phylogenetically more closely related to BSMV than to LRSV.

The results of our host range studies agreed with those of previous experiments, suggesting that PSLV is restricted to monocot hosts and differs from BSMV and LRSV in this regard. Positive-strand RNA viruses usually remodel the endomembrane systems to build replication factories for viral replication (Jin et al. 2018a). Our studies have recently shown that BSMV $\gamma$ b proteins are targeted to chloroplast replication sites, where they participate directly in viral replication by acting as a helicase enhancer during replication (Zhang et al. 2017). Hence, we speculated that PSLV $\gamma$ b may have similar enhancer properties; because the protein localizes to peroxisomes (Yelina et al. 2005), we questioned whether peroxisomes might be sites of PSLV replication.

Because $\gamma b$ has a functional role in the replication of BSMV, we performed experiments to determine whether the PSLV oa protein is also associated with peroxisomes. For the initial tests, we constructed $\alpha \mathrm{a}-\mathrm{GFP}$ fusions and found that the PSLV oa proteins localize to peroxisomes in agroinfiltrated $N$. benthamiana leaves. In contrast, both BSMV and LRSV oa-GFP were associated with chloroplasts, as we have previously established (Jiang et al. 2018; Jin et al. 2018b). We also found that PSLV and BSMV infections elicit similar ultrastructural changes in barley peroxisomes and chloroplasts, respectively, and that the observed anomalies include organelle degradation and membrane-derived vesicles (Fig. 3C). In addition, virus-like particles typical of PSLV were associated with the peroxisomes; in some instances, virus-like particles similar to PSLV virions radiated from the surface of the peroxisomes (Fig. 3C). These results provided evidence suggesting that PSLV replication occurs in association with peroxisomes rather than chloroplasts, as is the case with BSMV and LRSV, and suggested that one mechanism targeting viral replication proteins to different organelles may occur at the time of the initial establishment of hordeivirus replication sites. Future experiments are being planned to evaluate the extent to which the $\alpha$ a protein interacts with the $\gamma \mathrm{a}$ and $\gamma \mathrm{b}$ proteins and the gRNAs to form functional RdRps during PSLV replication and to identify PSLV and host interactions that establish replication sites during the early phases of PSLV infection.

Interestingly, despite the similar monocot hosts shared between PSLV and BSMV, the two viruses appear to select different organelles for the assembly of replication factories. Hence, our study emphasized that evaluating the replication of viruses in the same genus can provide very important information about virushost interactions during the early stages of infection and the possible novel interactions of $\mathrm{RdRp}$ proteins and gRNAs during the formation of replication factories (Jin et al. 2018a; Rubino and Russo 1998).

Despite the similarity among different hordeivirus $\gamma \mathrm{b}$ proteins (Fig. 1B), our results showed that the $\gamma \mathrm{b}$ derivatives vary in their VSR activities. BSMV $\gamma$ b elicited the strongest silencing suppressor activities, whereas the PSLV and LRSV had weaker suppressor functions (Fig. 4). Amino acid sequence alignments and mutagenesis showed that arginine and lysine residues in the basic motif are essential for RNA binding activities of BSMV $\gamma b$ (Donald and Jackson 1996). Both arginine and lysine residues are prevalent in the hordeivirus BM motif of $\gamma b$ proteins, but PSLV and LRSV $\gamma b$ have substantial differences from BSMV $\gamma \mathrm{b}$ within this region (Fig. 1B); however, whether these differences contribute to differences in VSR activities requires additional experiments. The coiled-coil motif in the $\mathrm{C}$-terminal region of the BSMV $\gamma \mathrm{b}$ protein is required 
for suppression of BSMV RNA silencing (Bragg and Jackson 2004), but the C-terminal amino acids of BSMV, LRSV, and PSLV $\gamma \mathrm{b}$ proteins differ in sequence and in the presence of SKL or SK Cterminal residues (Fig. 1B).
Our experiments revealed that the BSMV, LRSV, and PSLV $\gamma b$ proteins ectopically expressed in $N$. benthamiana leaves differ substantially in their silencing suppressor activities, with LRSV exhibiting the weakest suppression activities in the infiltration

A

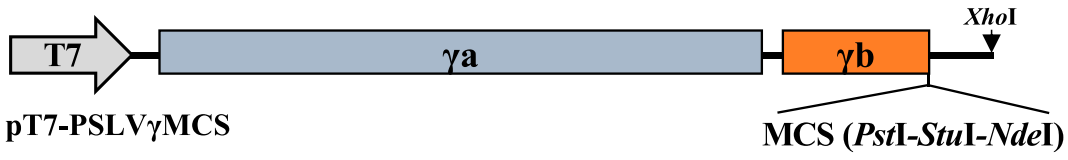

B
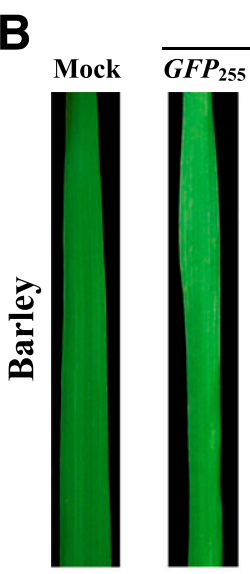

$\alpha+\beta+\gamma-$

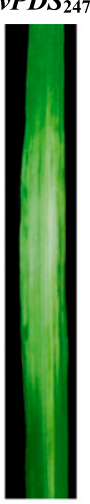

D
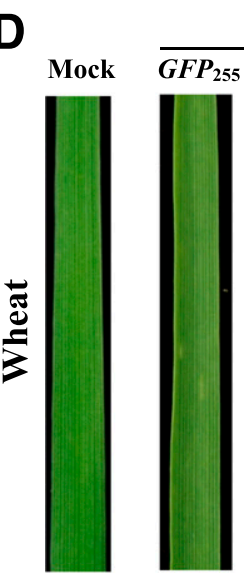

$\boldsymbol{\alpha}+\boldsymbol{\beta}+\boldsymbol{\gamma}-$
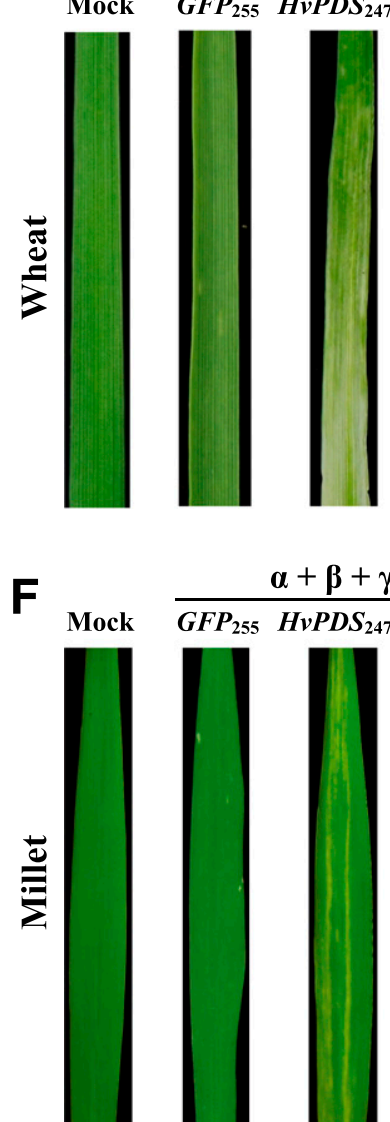

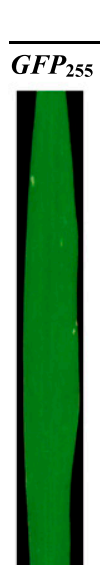

$\alpha+\beta+\gamma-$

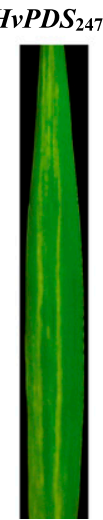

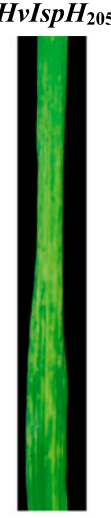

$\mathrm{HvIspH}_{205}$

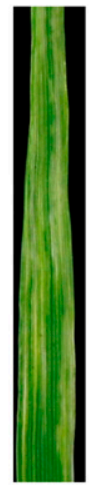

$\mathrm{HvIspH}_{205}$

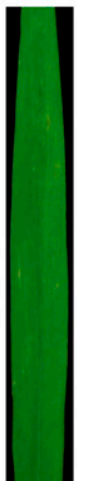

\section{C}
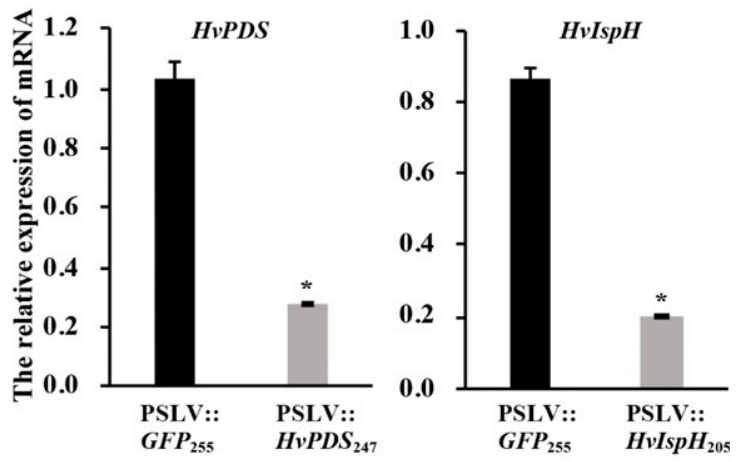

E
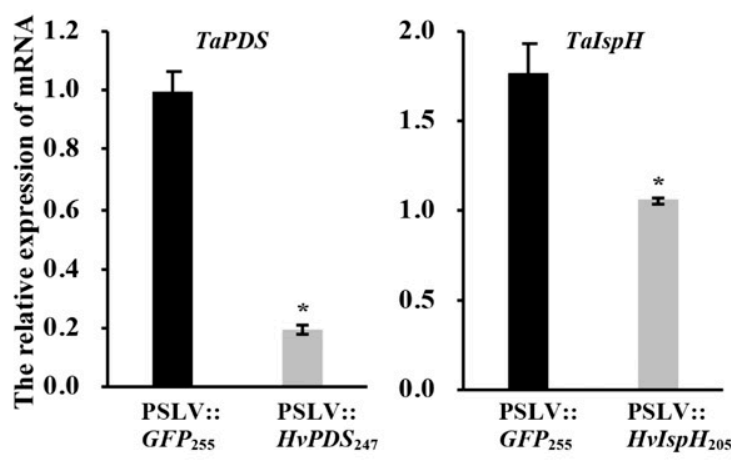

G
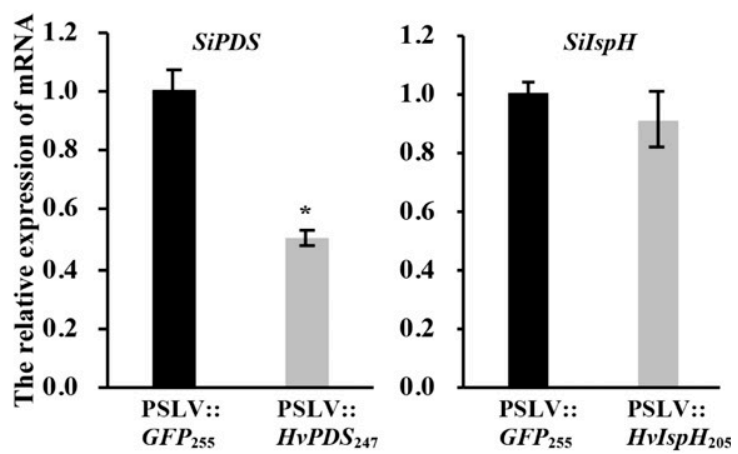

Fig. 7. Development of Poa semilatent virus (PSLV)-based virus-induced gene silencing (VIGS) for silencing barley, wheat, and millet genes. A, Schematic representation of the PSLV VIGS vector containing a multi-cloning site downstream of the PSLV $\gamma \mathrm{b}$ open reading frame (ORF) stop codon. XhoI indicates the restriction enzymes used for plasmid linearization. B to G, Evaluation of PSLV-based VIGS for silencing target genes in barley, wheat, and millet. PSLV::GFP 255 , PSLV::HvPDS 247 , and PSLV::HvIspH 205 were mechanically inoculated onto B, barley, D, wheat, and F, millet. Mock-inoculated plants served as controls. Systemic silencing phenotypes in the inoculated plants were documented at 15 days postinoculation (dpi). Experiments were repeated at least two times; representative results are shown. Quantitative reverse-transcription PCR (qRT-qPCR) analyses were conducted to evaluate changes in $P D S$ and $I s p H$ mRNA levels in plants inoculated with in vitro transcripts of various PSLV-derived VIGS constructs. Results shown in $\mathbf{C}, \mathbf{E}$, and $\mathbf{G}$ correspond to the qRT-PCR analyses shown in $\mathbf{B}$, D, and F. PSLV::GFP 255 -inoculated plants served as controls. Actin was used as a reference gene for data normalization. Error bars represent standard deviation from the mean $(n=3)$. *Statistical significance $(P<0.05)$ compared with the PSLV::GFP 255 control. 
assays (Fig. 4). In addition, the presence of the SKL peroxisometargeting signal resulted in obvious effects on silencing activity. For example, in leaf infiltration experiments, all three ectopically expressed $\gamma b$ proteins containing SKL motifs exhibited attenuated silencing suppressor activities over $\gamma \mathrm{b}$ proteins with C-terminal SK residues. (Fig. 6A to C). Although wheat infected with PSLV and the mutant PSLV ${ }_{\gamma \mathrm{b}-S K}$ derivatives exhibited similar latent phenotypes, the wild-type PSLV derivative replicated to higher levels than the mutant PSLV $\mathrm{V}_{\gamma \mathrm{b}-\mathrm{SK}}$ virus, as demonstrated by higher CP abundance in systemically infected leaves (Fig. 6D and E). Similar replication trends were observed in BSMV and LRSV systemically infected $N$. benthamiana leaves. In both cases, higher accumulation of $\mathrm{CP}$ occurred in leaves containing the BSMV and LRSV $\gamma \mathrm{bSKL}$ derivatives, suggesting that the attenuated silencing suppression activities of $\gamma b_{S K L}$ derivatives correlate with increased viral accumulation during the early stages of infection.

Observations that increased silencing suppressor activities of VSR proteins contributed to high levels of virus replication were consistent in numerous virus studies, but $\gamma b$ seems to differ from most common VSR proteins in this regard. It is of interest that the $\gamma b_{S K L}$ derivatives have reduced RNA silencing activities compared with those of the $\gamma b_{S K}$ derivatives. However, the mechanisms whereby VSR activities are implemented are only beginning to become evident. For example, the timing of VSR protein expression may affect various silencing activities and their effects on virus replication. For example, $\gamma \mathrm{b}$ protein expression, like many VSR proteins, is asynchronous during replication and lags behind the expression of the RdRp complex proteins. However, VSR derivatives may differ in their turnover rates and targeting to organelles that provide sites for replication or alternative sites that affect pathogenesis. Although we only have a vague idea about how such factors affect the abundance or targeting of $\gamma \mathrm{b}$ derivatives, $\gamma \mathrm{b}$ has several functions that have only recently been revealed. For example, our previous studies have shown that one of the multifunctional functions of the $\gamma \mathrm{b}$ protein is to enhance RNA unwinding of dsRNAs early during the infection cycle (Zhang et al. 2017), but the extent to which the $\gamma b_{S K L}$ and $\gamma b_{S K}$ derivatives affect this activity has not been investigated. Moreover, SKL targeting of the $\gamma b$ protein to peroxisomes could mitigate or sequester $\gamma b$ binding to small interfering RNAs that are usually produced in the cytoplasm during defense responses (Matzke et al. 2001). In addition, targeting of the $\gamma b_{\mathrm{SKL}}$ derivative to peroxisomes could facilitate activities of glycolate oxidase (GOX), a peroxisomeassociated flavin mononucleotide-containing enzyme that facilitates virus infection by suppressing peroxisomal ROS production (Yang et al. 2018b). Hence, we presumed that SKL-mediated targeting of BSMV $\gamma \mathrm{b}$ to the peroxisome may affect its positive roles in BSMV replication in ways that merit further studies. However, it should be pointed out that the increased peroxisome localization of $\gamma b$ may further inhibit the enzymatic activity of GOX and attenuate ROS-mediated plant defense responses, which might be more favorable for BSMV infection. This could be reflected, at least to some extent, by the higher $\mathrm{CP}$ accumulation of mutant $\mathrm{BSMV}_{\gamma \mathrm{b}-\mathrm{SKL}}$. Further experiments need to be performed to address how $\gamma \mathrm{b}$ functions coordinate to balance RNA silencing suppression, virus replication, and relevant disease phenotypes.

Viruses that cause severe symptoms in host plants are unsuitable VIGS vectors because the symptoms mask the silencing phenotypes (Igarashi et al. 2009). Hence, viruses that elicit mild infection phenotypes have an intrinsic advantage in the development of VIGS vectors (Burch-Smith et al. 2004; Igarashi et al. 2009). In this study, the mild phenotype of the cloned Canada isolate permitted engineering of PSLV as a new VIGS vector for cereal hosts. PSLVbased VIGS induces very mild symptoms, but the PDS VIGS vector efficiently suppressed $P D S$ expression in barley, wheat, and millet (Fig. 7B to G). We observed that PSLV::HvIspH ${ }_{205^{-}}$ inoculated wheat and barley developed an albino phenotype (Fig. 7B and D), whereas obvious silencing of $I s p H$ was not

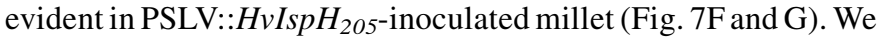
attributed this anomaly to the use of the HvIspH sequence for silencing because the HvIspH sequence shares only $85 \%$ nucleotide sequence identity with the millet SiIspH gene (Supplementary Fig. S5A), in contrast to the high nucleotide sequence identity ( $>91 \%$ ) shared among the HvPDS, TaPDS, and SiPDS genes (Supplementary Fig. S5B). The PSLV silencing vectors infect several hosts that have not been previously suitable for VIGS. It is worth pointing out that, in this regard, only Foxtail mosaic virus (FoMV) has been developed as a VIGS tool for foxtail millet (Liu et al. 2016). Hence, the PSLV-based VIGS tool developed in our study provides an additional alternative for gene function studies of several valuable crops.

In conclusion, we have constructed infectious cDNA clones from PSLV gRNAs and used these clones for comparisons with recombinant genomes of the BSMV and LRSV hordeiviruses. PSLV infection induces dramatic structural changes in peroxisomes, which we believe are PSLV replication sites. Different hordeivirus $\gamma \mathrm{b}$ proteins have varied VSR activities and differential effects on the symptom severity induced by BSMV hybrid viruses. The C-terminal SKL peroxisome-targeting signal has negative effects on $\gamma \mathrm{b}$ VSR activity, but it affects the disease phenotype of hordeiviruses to different extents. Our results enhance our understanding of the relatedness, pathogenesis, and replication of the hordeiviruses and provide an alternative tool for gene function studies in model monocots and economically important monocots such as barley, wheat, and millet.

\section{ACKNOWLEDGMENTS}

We thank Xianbing Wang, Jialin Yu, Chenggui Han, and Ying Wang at China Agricultural University for helpful suggestions during the progress of these experiments.

\section{LITERATURE CITED}

Agranovsky, A. A., Karasev, A. V., Novikov, V. K., Lunina, N. A., Loginov, S., and Tyulkina, L. G. 1992. Poa semilatent virus, a hordeivirus having no internal polydisperse poly(A) in the $3^{\prime}$ non-coding region of the RNA genome. J. Gen. Virol. 73:2085-2092.

Bragg, J. N., and Jackson, A. O. 2004. The C-terminal region of the Barley stripe mosaic virus $\gamma \mathrm{b}$ protein participates in homologous interactions and is required for suppression of RNA silencing. Mol. Plant Pathol. 5: 465-481.

Bragg, J. N., Lawrence, D. M., and Jackson, A. O. 2004. The N-terminal 85 amino acids of the Barley stripe mosaic virus $\gamma \mathrm{b}$ pathogenesis protein contain three zinc-binding motifs. J. Virol. 78:7379-7391.

Brakke, M. K., and Palomar, M. K. 1976. Separation of components of barley stripe mosaic virions by density-gradient centrifugation. Virology 71: 255-261.

Burch-Smith, T. M., Anderson, J. C., Martin, G. B., and Dinesh-Kumar, S. P. 2004. Applications and advantages of virus-induced gene silencing for gene function studies in plants. Plant J. 39:734-746.

Donald, R. G. K., and Jackson, A. O. 1994. The Barley stripe mosaic virus $\gamma \mathrm{b}$ gene encodes a multifunctional cysteine-rich protein that affects pathogenesis. Plant Cell 6:1593-1606.

Donald, R. G. K., and Jackson, A. O. 1996. RNA-binding activities of Barley stripe mosaic virus $\gamma \mathrm{b}$ fusion proteins. J. Gen. Virol. 77:879-888.

Edwards, M. C. 1995. Mapping of the seed transmission determinants of Barley stripe mosaic virus. Mol. Plant-Microbe Interact. 8:906-915.

Goodin, M. M., Dietzgen, R. G., Schichnes, D., Ruzin, S., and Jackson, A. O. 2002. pGD vectors: versatile tools for the expression of green and red fluorescent protein fusions in agroinfiltrated plant leaves. Plant J. 31: 375-383.

Hu, Y., Li, Z., Yuan, C., Jin, X., Yan, L., Zhao, X., Zhang, Y., Jackson, A. O., Wang, X., Han, C., Yu, J., and Li, D. 2015. Phosphorylation of TGB1 by protein kinase $\mathrm{CK} 2$ promotes Barley stripe mosaic virus movement in monocots and dicots. J. Exp. Bot. 66:4733-4747.

Huang, C., Qian, Y., Li, Z., and Zhou, X. 2012. Virus-induced gene silencing and its application in plant functional genomics. Sci. China Life Sci. 55:99-108.

Hunter, B. G., Heaton, L. A., Bracker, C. E., and Jackson, A. O. 1986. Structural comparison of Poa semilatent virus and Barley stripe mosaic virus. Phytopathology 76:322-326. 
Hunter, B. G., Smith, J., Fattouh, F., and Jackson, A. O. 1989. Relationship of Lychnis lingspot virus to Barley stripe mosaic virus and Poa semilatent virus. Intervirology 30:18-26.

Igarashi, A., Kousuke, Y., Tomokazu, S., Yukari, T., Emiko, S., Akihiro, T., Hajime, Y., Noriko, Y., Tsubasa, T., Masamichi, I., Hideki, T., and Nobuyuki, Y. 2009. Apple latent spherical virus vectors for reliable and effective virusinduced gene silencing among a broad range of plants including tobacco, tomato, Arabidopsis thaliana, cucurbits, and legumes. Virology 386:407-416.

Jackson, A. O., Lim, H.-S., Bragg, J., Ganesan, U., and Lee, M. Y. 2009. Hordeivirus replication, movement, and pathogenesis. Annu. Rev. Phytopathol. 47:385-422.

Jiang, Z., Li, Z., Yue, N., Zhang, K., Li, D., and Zhang, Y. 2018. Construction of infectious clones of Lychnis ringspot virus and evaluation of its relationship with Barley stripe mosaic virus by reassortment of genomic RNA segments. Virus Res. 243:106-109.

Jin, X., Cao, X., Wang, X., Jiang, J., Wan, J., Laliberte, J. F., and Zhang, Y. 2018a. Three-dimensional architecture and biogenesis of membrane structures associated with plant virus replication. Front. Plant Sci. 9:57.

Jin, X., Jiang, Z., Zhang, K., Wang, P., Cao, X., Yue, N., Wang, X., Zhang, X., Li, Y., Li, D., Kang, B. H., and Zhang, Y. 2018b. Three-dimensional analysis of chloroplast structures associated with virus infection. Plant Physiol. 176:282-294.

Johansen, L. K., and Carrington, J. C. 2001. Silencing on the spot. Induction and suppression of RNA silencing in the Agrobacterium-mediated transient expression system. Plant Physiol. 126:930-938.

Johnson, J. A., Bragg, J. N., Lawrence, D. M., and Jackson, A. O. 2003. Sequence elements controlling expression of Barley stripe mosaic virus subgenomic RNAs in vivo. Virology 313:66-80.

Lee, M. Y., Yan, L., Gorter, F. A., Kim, B. Y. T., Cui, Y., Hu, Y., Yuan, C., Grindheim, J., Ganesan, U., Liu, Z., Han, C., Yu, J., Li, D., and Jackson, A. O. 2012. Brachypodium distachyon line $\mathrm{Bd} 3-1$ resistance is elicited by the Barley stripe mosaic virus triple gene block 1 movement protein. J. Gen. Virol. 93:2729-2739.

Liu, D., Shi, L., Han, C., Yu, J., Li, D., and Zhang, Y. 2012. Validation of reference genes for gene expression studies in virus-infected Nicotiana benthamiana using quantitative real-time PCR. PLoS One 7:e46451.

Liu, N., Xie, K., Jia, Q., Zhao, J., Chen, T., Li, H., Wei, X., Diao, X., Hong, Y., and Liu, Y. 2016. Foxtail mosaic virus-induced gene silencing in monocot plants. Plant Physiol. 171:1801-1807.

Llave, C., Kasschau, K. D., and Carrington, J. C. 2000. Virus-encoded suppressor of posttranscriptional gene silencing targets a maintenance step in the silencing pathway. Proc. Natl. Acad. Sci. USA 97:13401-13406.

Matzke, M. A., Matzke, A. J. M., Pruss, G. J., and Vance, V. B. 2001. RNAbased silencing strategies in plants. Curr. Opin. Genet. Dev. 11:221-227.

Nelson, B. K., Cai, X., and Nebenführ, A. 2007. A multicolored set of in vivo organelle markers for co-localization studies in Arabidopsis and other plants. Plant J. 51:1126-1136.

Petty, I. T. D., Hunter, B. G., Wei, N., and Jackson, A. O. 1989. Infectious Barley stripe mosaic virus RNA transcribed in vitro from full-length genomic cDNA clones. Virology 171:342-349.

Polák, Z., and Slykhuis, J. T. 1972. Comparisons of poa semilatent and barley stripe mosaic viruses. Can. J. Bot. 50:263-267.

Rubino, L., and Russo, M. 1998. Membrane targeting sequences in tombusvirus infections. Virology 252:431-437.
Savenkov, E. I., Solovyev, A. G., and Morozov, S. Y. 1998. Genome sequences of poa semilatent and lychnis ringspot hordeiviruses. Arch. Virol. 143: 1379-1393.

Slykhuis, J. T. 1972. Poa semilatent virus from native grasses. Phytopathology 62:508-513.

Solovyev, A. G., Savenkov, E. I., Agranovsky, A. A., and Morozov, S. Y. 1996. Comparisons of the genomiccis-elements and coding regions in RNA $\beta$ components of the hordeiviruses Barley stripe mosaic virus, Lychnis ringspot virus, and Poa semilatent virus. Virology 219:9-18.

Solovyev, A. G., Savenkov, E. I., Grdzelishvili, V. Z., Kalinina, N. O., Morozov, S. Y., Schiemann, J., and Atabekov, J. G. 1999. Movement of hordeivirus hybrids with exchanges in the triple gene block. Virology 253: 278-287.

Verchot-Lubicz, J., Torrance, L., Solovyev, A. G., Morozov, S. Y., Jackson, A. O., and Gilmer, D. 2010. Varied movement strategies employed by triple gene block-encoding viruses. Mol. Plant-Microbe Interact. 23: 1231-1247.

Yang, H., Shi, Y., Liu, J., Guo, L., Zhang, X., and Yang, S. 2010. A mutant CHS3 protein with TIR-NB-LRR-LIM domains modulates growth, cell death and freezing tolerance in a temperature-dependent manner in Arabidopsis. Plant J. 63:283-296.

Yang, M., Li, Z., Zhang, K., Zhang, X., Zhang, Y., Wang, X., Han, C., Yu, J., $\mathrm{Xu}, \mathrm{K}$., and Li, D. 2018b. Barley stripe mosaic virus gammab interacts with Glycolate Oxidase and inhibits peroxisomal ROS production to facilitate virus infection. Mol. Plant 11:338-341.

Yang, M., Zhang, Y., Xie, X., Yue, N., Li, J., Wang, X. B., Han, C., Yu, J., Liu, Y., and Li, D. 2018a. Barley stripe mosaic virus gammab protein subverts autophagy to promote viral infection by disrupting the ATG7-ATG8 interaction. Plant Cell 30:1582-1595.

Yao, M., Zhang, T., Tian, Z., Wang, Y., and Tao, X. 2011. Construction of Agrobacterium-mediated Cucumber mosaic virus infectious cDNA clones and $2 \mathrm{~b}$ deletion viral vector. Scientia Agricultura Sinica 44:3060-3068.

Yelina, N. E., Erokhina, T. N., Lukhovitskaya, N. I., Minina, E. A., Schepetilnikov, M. V., Lesemann, D.-E., Schiemann, J., Solovyev, A. G., and Morozov, S. Y. 2005. Localization of Poa semilatent virus cysteine-rich protein in peroxisomes is dispensable for its ability to suppress RNA silencing. J. Gen. Virol. 86:479-489.

Yelina, N. E., Savenkov, E. I., Solovyev, A. G., Morozov, S. Y., and Valkonen, J. P. T. 2002. Long-distance movement, virulence, and RNA silencing suppression controlled by a single protein in Hordei- and Potyviruses: Complementary functions between virus families. J. Virol. 76:12981-12991.

Yuan, C., Li, C., Yan, L., Jackson, A. O., Liu, Z., Han, C., Yu, J., and Li, D. 2011. A high throughput Barley stripe mosaic virus vector for virus induced gene silencing in monocots and dicots. PLoS One 6:e26468.

Zhang, K., Zhang, Y., Yang, M., Liu, S., Li, Z., Wang, X., Han, C., Yu, J., and Li, D. 2017. The Barley stripe mosaic virus gammab protein promotes chloroplast-targeted replication by enhancing unwinding of RNA duplexes. PLoS Pathog 13:e1006319.

Zhang, X., Dong, K., Xu, K., Zhang, K., Jin, X., Yang, M., Zhang, Y., Wang, X., Han, C., Yu, J., and Li, D. 2018. Barley stripe mosaic virus infection requires PKA-mediated phosphorylation of gammab for suppression of both RNA silencing and the host cell death response. New Phytol. 218: 1570-1585. 